\title{
Paramorphic multicarrier communications for interference mitigation
}

\author{
Matt Carrick ${ }^{*}$ (D), Jeffrey H. Reed ${ }^{1}$ and Chad M. Spooner ${ }^{2}$
}

\begin{abstract}
This paper presents a novel method for enabling communication in cyclostationary interference limited environments by adaptively inserting and exploiting spectral redundancy in an orthogonal frequency division multiplexing (OFDM) signal. The redundancy is formed through repeating data symbols across OFDM symbols in both time and frequency. A novel frequency shift (FRESH) filter is applied to exploit the time-varying cyclostationary properties, enabling the communication while under interference.

The ability to design an interference mitigating property into the communication signal creates a new degree of freedom when optimizing and adapting a waveform's parameters. Simulation results demonstrate the performance gains in both signal to interference and noise ratio (SINR) and bit error rate (BER) as compared to traditional time-invariant optimal filtering techniques and error correcting codes (ECC).
\end{abstract}

Keywords: FRESH filter, Cyclostationary, Spectral redundancy, Interference mitigation, Waveform optimization, Paramorphic waveform

\section{Introduction}

This paper proposes a method where spectral redundancy is adaptively designed into a multicarrier signal for communicating in cyclostationary (CS) interference limited environments. Investing into redundancies within a signal to produce a gain at the receiver is a common trade-off in wireless communication system design. Common examples of this trade-off include trading bandwidth for robustness by using coding to improve the bit error rate and using a larger cyclic prefix on an orthogonal frequency division multiplexing (OFDM) signal to improve the signal to noise ratio (SNR) at the receiver. These two solutions overcome traditional problems in wireless communications: communicating in noise limited environments and the affects of multipath channels. This paper focuses on the separate but related problem of communicating within cyclostationary interference limited environments, where other communication signals are directly in-band of the desired signal. Simulation results demonstrate that under the given interference scenarios, transmitting redundant spectra at selected positions in the overall spectrum and

*Correspondence: mcarrick@vt.edu

${ }^{1}$ Bradley Department of Electrical and Computer Engineering, Virginia

Polytechnic and State University, Blacksburg, Virginia, 24061, USA

Full list of author information is available at the end of the article optimally combining it at the receiver enables reliable communication which is otherwise not possible using error correcting codes alone.

The novelty of the proposed method includes:

- Implements a method for communicating in cyclostationary interference limited environments.

- Adaptable and dynamic and adjusts the waveform parameters and locations of redundancies to the spectral environment on the fly.

- Implements a novel filter for exploiting time-varying cyclostationary properties of signals.

- Provides more than $16.5 \mathrm{~dB}$ worth of SNR gain at a BER of $10^{-5}$ within the given simulation as compared to low density parity check (LDPC) error correcting codes.

The proposed method introduces redundancies into the signal, analogous to an error correcting code (ECC). The proposed method is explicitly designed for cyclostationary interference, in direct contrast to ECCs which are designed for stationary noise. The performance of ECCs degrades quickly under wideband cyclostationary inter- 
ference because these assumptions are not valid; however, the paramorphic waveform is much more robust under the same conditions because it is specifically designed for the signal environment at hand.

A FRESH filter is applied to mitigate the interference and collapse the expanded bandwidth down to its nominal value, analogous to a direct-sequence spread spectrum (DSSS) receiver. The bandwidth expansion of the DSSS signal is fixed, a function of the spreading sequence. The distribution of the signal energy is inefficient as it is smeared across the spectrum due to the spreading operation, requiring bandwidth expansion factors of 15 or 31 for short code DSSS [1]. The proposed technique only requires a bandwidth expansion factor of $K$ to mitigate the impact of $K-1$ full bandwidth interferers because the redundancies are optimally combined at the receiver [2]. The method allows for more flexibility, placing redundancies across the spectrum with finer detail and adapting their location and power levels according to the interference.

Simulated annealing is used to optimize the waveform parameters in light of the interference rejection capability, balancing the improved BER with the reduction in spectral efficiency, approximating an optimal solution to a non-linear multi-objective optimization problem.

Multicarrier waveforms designed with spectral redundancy are referred to as paramorphic ${ }^{1}$ multicarrier waveforms (PMW), requiring new adaptive demodulators to incorporate the redundancy into the estimate of the desired signal, referred to as paramorphic FRESH demodulators (PFD).

\subsection{Related work}

A comparison between various interference mitigation techniques and FRESH filtering is described, followed by the related contributions within FRESH filtering, and finally, the contributions of the proposed method are described in relation to the existing literature.

FRESH filtering occupies two overlapping, but distinct fields: adaptive filtering and cyclostationary signal processing. The FRESH filter exploits the cyclostationary properties of signals and does so through the use of adaptive periodically time varying (PTV) filtering, making it well suited for interference mitigation [2]. It can be considered as supplementing existing methods, as it is a more general case of the optimal time invariant (TI) filter [2,3]. FRESH filters, being PTV filters, outperform their TI counterparts at the cost of additional filtering complexity. As FRESH filters exploit spectral redundancies, they are broadly applicable. This includes further improving the interference mitigation properties of DSSS [4] and array processing systems [5]. A more in-depth discussion of the advantages and disadvantages of FRESH filtering can be found in $[6,7]$.
There has been much research on CS signals and their processing [6] with the most recent interest being in the applications to OFDM and multicarrier signals [7]. The theory of optimal periodically time-varying filtering for CS signals was proposed in [8], which would later become known as FRESH filtering [2]. Frequency-domain FRESH filtering was developed in [9] and the adaptation of such structures in $[10,11]$. Improving the CS features of OFDM signals has been proposed in $[12,13]$ for network identification and cognitive radio applications. In [13, 14], the cyclostationarity of an OFDM signal is incorporated by repeating symbols in frequency and time, respectively, for the purpose of user network identification. The use of FRESH filtering to exploit the spectral redundancy of CS OFDM signals is suggested in [15] but not explored any further. The use of FRESH filtering to exploit the spectral redundancy in OFDM signals induced by the cyclic prefix is proposed in $[16,17]$.

A time-varying FRESH (TV-FRESH) filter was first presented in [18] to exploit time-varying cyclostationary in OFDM signals and mitigate wideband interference, while [19] demonstrated the applicability for improving equalization in generalized frequency division multiplexing (GFDM) signals [19]. The TV-FRESH filter was also used in [20] for mitigating the interference radar signals to OFDM.

This paper distinguishes with respect to the existing literature itself by presenting a system for designing and adapting the cyclostationary properties of OFDM signals for interference mitigation. The paramorphic method expands upon $[2,8-11,16,17]$ by proposing a frequency domain filter which exploits time-varying cyclostationary features. Additionally, the proposed method utilizes the cyclostationarity of OFDM for improving the robustness of communications, where [12-14] focused on network identification.

\subsection{Outline}

The remainder of the paper is outlined as follows. Section 2 provides relevant background information on cyclostationary signals, FRESH filtering, and simulated annealing. In Section 3, the proposed approach is described, including the signal model, improved demodulator and its optimal weights, and the optimization of the waveform design and demodulator. Section 4 presents simulation results and a complexity analysis, and Section 5 concludes the paper.

\section{Background}

The proposed method in Section 3 presents a novel FRESH filter for exploiting spectral redundancy within CS signals and jointly optimizes the waveform and demodulator using simulated annealing. The model for the received signal is described in Section 2.1. Cyclostationary signals 
and their relevant properties are described in Section 2.2. The FRESH filtering structure and the derivation of its minimum mean squared error (MMSE) filter weights is given in Section 2.3, and an introduction to simulated annealing is presented in Section 2.4.

\subsection{Received signal model}

The received signal model used within this paper is described by:

$$
x(t)=(d(t) \otimes \phi(t))+(\psi(t) \otimes i(t))+n(t),
$$

where $x(t)$ is the received signal, $d(t)$ is the desired signal and its channel is $\phi(t), i(t)$ is the CS interference and its channel is $\psi(t)$, and $n(t)$ is the stationary white noise. The convolution operator is represented by $\otimes$, defined as:

$$
a(t) \otimes b(t)=\int_{-\infty}^{\infty} a(\tau) b(t-\tau) d \tau .
$$

The analytic expressions in the remainder of this paper often use the frequency domain representation, which is given by:

$$
X(f)=D(f) \Phi(f)+I(f) \Psi(f)+N(f),
$$

where $X(f)=\mathcal{F}\{x(t)\}$.

\subsection{Cyclostationary signals}

Cyclostationary signals are those which contain secondorder periodicity in the time domain and spectral redundancy in the frequency domain. The spectral correlation density function, $S_{x}^{\alpha}(f)$, given in (4) [21], is a measure of spectral redundancy at CF $\alpha[2]$, and $X_{T}(t, f)$ is the shorttime Fourier transform (5) of the signal $x(t)$. A CF is a relative difference in frequency for which the signal $x(t)$ spectrally correlates with itself, $S_{x}^{\alpha}(f) \neq 0$,

$$
\begin{aligned}
S_{x}^{\alpha}(f)= & \lim _{T \rightarrow \infty \Delta t \rightarrow \infty} \lim _{T \rightarrow \infty} \frac{1}{T \Delta t} \\
& \cdot \int_{-\frac{\Delta t}{2}}^{\frac{\Delta t}{2}} X_{T}\left(t, f+\frac{\alpha}{2}\right) X_{T}^{*}\left(t, f-\frac{\alpha}{2}\right) d t, \\
X_{T}(t, v)= & \int_{t-\frac{T}{2}}^{t+\frac{T}{2}} x(u) e^{-j 2 \pi v u} d u .
\end{aligned}
$$

Cyclostationary signals may also exhibit conjugate spectral correlation, $S_{x x^{*}}^{\beta}(f) \neq 0$, of which $\beta$ is a conjugate cycle frequency,

$$
\begin{aligned}
S_{x x^{*}}^{\beta}(f) & =\lim _{T \rightarrow \infty \Delta t \rightarrow \infty} \lim _{T \rightarrow \infty} \frac{1}{T \Delta t} \\
& \cdot \int_{-\frac{\Delta t}{2}}^{\frac{\Delta t}{2}} X_{T}\left(t, f+\frac{\beta}{2}\right) X_{T}\left(t,-f+\frac{\beta}{2}\right) d t .
\end{aligned}
$$

A signal is CS for any combination of $S_{x}^{\alpha}(f) \neq 0$ for $\alpha \neq$ 0 and $S_{x x^{*}}^{\beta}(f) \neq 0$. All finite-power signals have at least one CF, $\alpha=0$, and there may be multiple CFs present in one signal.

\subsection{FRESH filtering}

A FRESH filter is the optimal widely linear filter for CS signals [2]. It exploits spectrally redundant information in its input to produce an estimate of the desired signal. The received signal is filtered through a parallel set of FSs followed by linear time-invariant filters, and the results are summed. Both the spectral redundancy and conjugate spectral redundancies are exploited as they contain redundant information and should be used to produce a better estimate of the desired signal. The FRESH filter is defined in (7) [2], and $M$ and $N$ are the number of FSs corresponding to the spectral correlation and conjugate spectral correlation. The FRESH filter (7) is illustrated schematically in Fig. 1,

$$
\begin{aligned}
\breve{d}(t)= & \sum_{m=0}^{M-1} a_{m}(t) \otimes x(t) e^{j 2 \pi \alpha_{m} t} \\
& +\sum_{n=0}^{N-1} b_{n}(t) \otimes x^{*}(t) e^{j 2 \pi \beta_{n} t} .
\end{aligned}
$$

The set of FSs $\{\alpha\}$ can come from the desired signal $d(t)$ and CS interference $i(t)$ within the received signal $x(t)$. Linearly combining $d(t)$ and $i(t)$ creates additional second-order periodicities within $x(t)$ from their CF differences which can be exploited by the FRESH filter [2]. The set of CFs corresponding to the desired signal is given by $\left\{\gamma_{d}\right\}=\left\{\gamma_{d, 0}, \gamma_{d, 1}, \gamma_{d, 2}, \ldots\right\}$, and the set for the interference is given by $\left\{\gamma_{i}\right\}=\left\{\gamma_{i, 0}, \gamma_{i, 1}, \gamma_{i, 2}, \ldots\right\}$. The set of all FSs $\{\alpha\}$ is therefore:

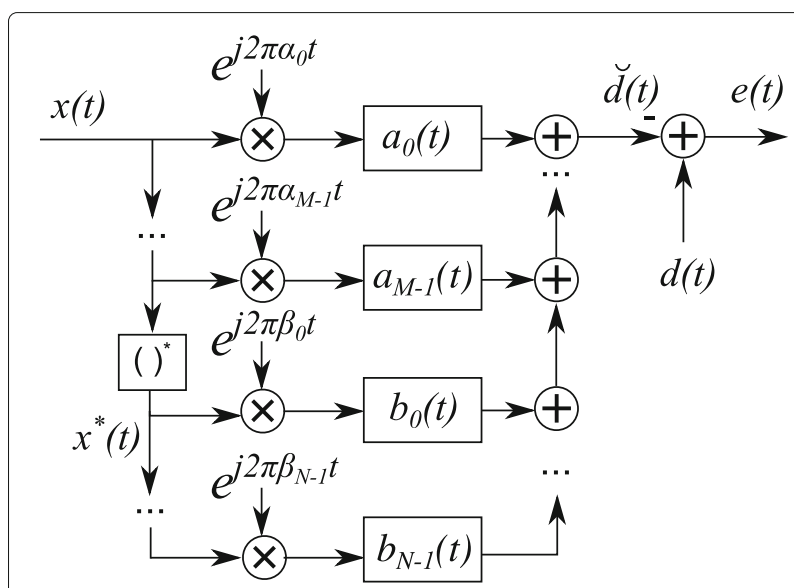

Fig. 1 The time-domain implementation of the FRESH filter from [2] 


$$
\begin{aligned}
\{\alpha\}= & \left\{\gamma_{d, 0}, \gamma_{d, 1}, \gamma_{d, 2}, \ldots,\right. \\
& \gamma_{i, 0}, \gamma_{i, 1}, \gamma_{i, 2}, \ldots, \\
& \gamma_{d, 0}-\gamma_{i, 0},-\gamma_{d, 0}+\gamma_{i, 0}, \\
& \gamma_{d, 0}-\gamma_{i, 1},-\gamma_{d, 0}+\gamma_{i, 1}, \\
& \ldots, \\
& \gamma_{d, 1}-\gamma_{i, 0},-\gamma_{d, 1}+\gamma_{i, 0}, \\
& \gamma_{d, 1}-\gamma_{i, 1},-\gamma_{d, 1}+\gamma_{i, 1}, \\
& \ldots\} .
\end{aligned}
$$

The FS $\alpha_{m}$ is therefore the $m^{\text {th }}$ element of the set $\{\alpha\}$, and $\{\alpha\}$ does not have to be ordered in a specific manner. The set $\{\beta\}$ is formed similarly. If $\left\{\zeta_{d}\right\}$ is the set of CFs of the conjugate spectral redundancy for the desired signal, and $\left\{\zeta_{i}\right\}$ for the interference, then:

$$
\begin{aligned}
\{\beta\}=\left\{\zeta_{d, 0}, \zeta_{d, 1}, \zeta_{d, 2}, \ldots,\right. & \ldots \\
& \zeta_{i, 0}, \zeta_{i, 1}, \zeta_{d, 2}, \ldots, \\
& \zeta_{d, 0}-\zeta_{i, 0},-\zeta_{d, 0}+\zeta_{i, 0}, \\
& \zeta_{d, 0}-\zeta_{i, 1},-\zeta_{d, 0}+\zeta_{i, 1}, \\
& \ldots, \\
& \zeta_{d, 1}-\zeta_{i, 0},-\zeta_{d, 2}+\zeta_{i, 0} \\
& \zeta_{d, 1}-\zeta_{i, 1},-\zeta_{d, 2}+\zeta_{i, 1}, \\
& \ldots\} .
\end{aligned}
$$

Both (8) and (9) represent the sets of all cycle frequencies corresponding to the spectral redundancy and conjugate spectral redundancy. Both the desired signal and interference can have multiple cycle frequencies present, and the received signal contains combinations of the sums and differences of the cycle frequencies. Thus, the sets (8) and (9) represent all of the cycle frequencies of the received signal.

The FSs $e^{j 2 \pi \alpha_{m} t}$ align the spectral redundancies within $x(t)$, which are then combined using the filters $a_{m}(t)$. Similarly, the FSs $e^{j 2 \pi \beta_{n} t}$ align the conjugate spectral redundancies within $x^{*}(t)$, which are combined using the filters $b_{n}(t)$.

One method for determining the optimal filters $a_{m}(t)$ and $b_{n}(t)$ is to minimize the mean squared error (MSE) produced by the FRESH filter, where the error is the difference between the desired signal $d(t)$ and its estimate, $\breve{d}(t)$. Finding the filter weights which minimize the MSE is simpler mathematically in the frequency domain; therefore, the filter estimate $\mathcal{F}\{\breve{d}(t)\}$ is transformed into the frequency domain in (10),

$$
\begin{aligned}
\breve{D}(f)= & \sum_{m=0}^{M-1} A_{m}(f) X\left(f-\alpha_{m}\right) \\
& +\sum_{n=0}^{N-1} B_{n}(f) X^{*}\left(-f+\beta_{n}\right),
\end{aligned}
$$

where $\mathcal{F}\left\{a_{m}(t)\right\}=A_{m}(f)$ and $\mathcal{F}\left\{b_{n}(t)\right\}=B_{n}(f)$. The first step in finding the filter weights corresponding to the MMSE is forming the filter error and then the MSE. The filter error $E(f)$ is given by (11), where $\mathcal{F}\{d(t)\}=D(f)$. The mean squared error $E_{M S E}(f)$ is given by (12),

$$
\begin{aligned}
& E(f)=D(f)-\breve{D}(f), \\
& E_{M S E}(f)=\mathbb{E}\left\{E(f) E^{*}(f)\right\} .
\end{aligned}
$$

The MSE of (12) can be found by taking its derivative and setting equal to zero. The derivative of the MSE is done with respect to the filter weights $A_{m}(f)$ and $B_{n}(f)$, the frequency domain versions of the filters in (7).

To find the filters $A_{m}(f)$ and $B_{n}(f)$ that minimize the MSE, the derivative of the MSE is taken with respect to both sets of filters and set equal to zero as in (13) and (14),

$$
\begin{aligned}
& \frac{\partial E_{\mathrm{MSE}}(f)}{\partial A_{m}^{*}(f)}=0, \\
& \frac{\partial E_{\mathrm{MSE}}(f)}{\partial B_{n}^{*}(f)}=0 .
\end{aligned}
$$

Performing the derivatives of the MSE results in two orthogonal projections as expected for a MMSE filter, (15) and (16). The projections state that the filter error $E(f)$ is orthogonal to the input $X(f)$ and $X^{*}(f)$ at each of the FSs,

$$
\begin{aligned}
& \mathbb{E}\left\{E(f) X^{*}\left(f-\alpha_{m}\right)\right\}=0, \\
& \mathbb{E}\left\{E(f) X\left(-f+\beta_{n}\right)\right\}=0 .
\end{aligned}
$$

The filter error $E(f)(11)$ is substituted into the orthogonal projections (15) and (16), resulting in the FRESH filter design equations in [2],

$$
\begin{aligned}
S_{d x}^{\alpha_{p}}\left(f-\frac{\alpha_{p}}{2}\right)= & \sum_{m=0}^{M-1} S_{x}^{\alpha_{p}-\alpha_{m}}\left(f-\frac{\alpha_{m}+\alpha_{p}}{2}\right) A_{m}(f) \\
& +\sum_{n=0}^{N-1} S_{x x^{*}}^{\beta_{n}-\alpha_{p}}\left(f-\frac{\beta_{n}+\alpha_{p}}{2}\right)^{*} B_{n}(f) \\
p=0,1, \ldots, M-1, & \\
S_{d x^{*}}^{\beta_{q}}\left(f-\frac{\beta_{q}}{2}\right)= & \sum_{m=0}^{M-1} S_{x x^{*}}^{\beta_{q}-\alpha_{m}}\left(f-\frac{\alpha_{m}+\beta_{q}}{2}\right) A_{m}(f) \\
& +\sum_{n=0}^{N-1} S_{x}^{\beta_{q}-\beta_{n}}\left(-f+\frac{\beta_{n}+\beta_{q}}{2}\right) B_{n}(f),
\end{aligned}
$$

$$
q=0,1, \ldots, N-1 \text {. }
$$

The Wiener filter is a degenerate case of the FRESH filter where $\{\alpha\}=\{0\}$ and $\{\beta\}=\varnothing$, where $\varnothing$ is the null set. In this case, no spectral redundancy is exploited by the filter leading to a decrease in performance relative to the FRESH filter. 


\subsection{Simulated annealing}

Simulated annealing [22] is a meta-heuristic optimization approach that has been applied to cognitive radio [23]. Simulated annealing derives its technique from metallurgy, where the temperature of a metal is high while being shaped and is gradually cooled as it takes its final shape. In our context, this process is simulated by testing a series of candidate solutions (heating and forging), measuring their performance, and gradually narrowing down the scope of potential solutions over time (cooling). To avoid focusing on a local maxima, simulated annealing uses a probabilistic function to determine if a solution with relatively worse performance will be accepted. Over time, this probability is decreased, allowing the algorithm to find the global maximum.

Simulated annealing is used in Section 3 to optimize the waveform parameters which minimize the BER while maximizing the spectral efficiency, subject to an objective function. The multi-objective non-linear optimization problem makes deriving an analytic solution difficult, and therefore, simulated annealing is used to approximate the optimal solutions.

\section{Paramorphic multicarrier waveform and demodulator}

We propose to insert spectral redundancy into an OFDM signal through data-symbol repetition to form a paramorphic multicarrier waveform. A paramorphic FRESH demodulator is then developed to exploit the time-varying spectral redundancy designed into the signal. A signal model is created in Section 3.1 to describe the nature of the symbol repetition, and the resulting CS properties are described in Section 3.2. The novel demodulator which exploits the time-varying spectral redundancy is proposed in Section 3.4 and its MMSE filter weights are derived in Section 3.5. The theoretical SINR at the output of the novel FRESH filter is derived in Section 3.6, and a joint optimization is proposed in Section 3.7 to select the best operating parameters of the new paramorphic waveform.

\subsection{Signal model}

The standard OFDM signal model is provided and adapted to include symbol repetition that creates exploitable spectral redundancy. An OFDM signal with $N$ subcarriers is represented by (19) and (20) in the time and frequency domains, respectively [13]:

$$
\begin{aligned}
& \tilde{d}(t)=\sum_{l=0}^{L-1} \sum_{n=0}^{N-1} a_{l, n} q(t-l T) e^{j 2 \pi \frac{n}{N} t}, \\
& \tilde{D}(f)=\sum_{l=0}^{L-1} \sum_{n=0}^{N-1} a_{l, n} Q\left(f-\frac{n}{N}\right) e^{-j 2 \pi\left(f-\frac{n}{N}\right) l T} .
\end{aligned}
$$

In these models $a_{l, n}$ is the data-symbol of the $l^{\text {th }}$ OFDM symbol applied to subcarrier $n$ and $q(t)$ is the rectangular pulse shape, where $Q(f)=\mathcal{F}\{q(t)\}$. $T$ is the length of the window, including the cyclic prefix time, where $T=T_{C P}+T_{s}$.

The OFDM model in (19) and (20) is modified by incorporating data-symbol repetition across both time and frequency, requiring block-based transmissions of $B$ OFDM symbols. The data-symbols to be transmitted within each block are represented by $a_{l, 0}, a_{l, 1}, \ldots, a_{l, M-1}$, and the $m^{\text {th }}$ symbol $a_{l, m}$ is repeated $R(m)$ times within the block. The data-symbol $a_{l, m}$ is mapped onto the subcarrier at frequency $f_{m, r}$ where $r=0,1, \ldots, R(m)-1$. The signal model for the $c^{\text {th }}$ OFDM symbol within the $l^{\text {th }}$ block for the proposed technique is therefore [18]:

$$
\begin{aligned}
d_{l, c}(t) & =\sum_{m=0}^{M-1} \sum_{r=0}^{R(m)-1} a_{l, m} q(t-(B l+c) T) e^{j 2 \pi f_{m, r} t} \\
D_{l, c}(f) & =\sum_{m=0}^{M-1} \sum_{r=0}^{R(m)-1} a_{l, m} Q\left(f-f_{m, r}\right) \\
& \cdot e^{-j 2 \pi\left(f-f_{m, r}\right)(B l+c) T} .
\end{aligned}
$$

The symbols $a_{l, n}$ of (19) are typically assumed to be independent and identically distributed (IID) [24, 25]. The symbols applied to the subcarriers are no longer independent, as correlation has been introduced into the signal by design in (21).

The signals over all time are therefore $d(t)=$ $\sum_{l} \sum_{c=0}^{B-1} d_{l, c}(t)$ and $D(f)=\sum_{l} \sum_{c=0}^{B-1} D_{l}(f)$.

\subsection{Cycle frequencies of paramorphic waveform}

The cycle frequencies of the received signal must be known to the receiver when constructing the PFD. As in (8) and (9), the cycle frequencies come from the desired signal and the interference. In this section, the cycle frequencies of the desired signal are described, while the cycle frequencies of the interference are described in Section 3.3.

The PMW is able to independently select any linear modulation format on each of its subcarriers; however, the type of modulation will have an impact on the cyclostationary properties of the signal. Formats such as quadrature amplitude modulation (QAM) and phase shift keying (PSK) will induce spectral redundancy when their symbols are repeated across the spectrum, while others such as pulse amplitude modulation (PAM) and binary phase shift keying (BPSK) in addition will induce conjugate spectral redundancy. The impact of these formats are highlighted here because they are commonly used, but it does not preclude the use of other constellations.

The CFs corresponding to the repetitions of symbol $a_{l, m}$ must be known to the receiver so the spectral redundancy, 
which is derived from the repetition patterns of the datasymbols, can be exploited. Examples of repetition patterns are given in Figs. 2 and 3.

While repeating data-symbols creates spectral redundancy, some modulations have inherent conjugate spectral redundancy which can also be exploited. The test for spectral redundancy is given by [26]:

$$
\mathbb{E}\left\{v v^{*}\right\} \neq 0,
$$

and the test for conjugate spectral redundancy is given by:

$$
\mathbb{E}\{v v\} \neq 0,
$$

where $v$ is the set of all constellation points within a modulation. A modulation format contains spectral redundancy when (23) is true and and conjugate spectral redundancy when (24) is true.

For QPSK, $v=\{-1-j,-1+j, 1-j, 1+j\}$, and from (23),

$$
\mathbb{E}\left\{|-1-j|^{2}+|-1+j|^{2}+|1-j|^{2}+|1+j|^{2}\right\} \neq 0,
$$

and according to (24),

$$
\mathbb{E}\left\{(-1-j)^{2}+(-1+j)^{2}+(1-j)^{2}+(1+j)^{2}\right\}=0,
$$

therefore, it only contains spectral redundancy. For BPSK, $\mathbb{E}\left\{(-1)^{2}+(1)^{2}\right\} \neq 0$ and $\mathbb{E}\left\{|-1|^{2}+|1|^{2}\right\} \neq 0$; thus, it has both spectral correlation and conjugate spectral correlation. Other modulations can be tested for spectral redundancy and conjugate spectral redundancy by applying (23) and (24). Once the type of redundancy is known, its cycle frequencies can be incorporated into the PFD as in (31) and (32).

The CFs are therefore defined according to the modulation and their location within the repetition pattern. As data-symbols can be repeated in time, new notation is needed to capture the now time-varying spectral redundancy. The notation $\gamma_{c, b, u}$ is described in Fig. 2, show- ing how the cycle frequencies align spectral redundancies in time. For example, $\gamma_{2,1, k}$ frequency shifts OFDM symbol 1 to align the redundancies in OFDM symbol 2. The $k$ index represents the $k^{\text {th }}$ cycle frequency between OFDM symbol 1 and 2. The same is true for $\zeta_{c, b, v}$ which represents the CFs of the conjugate spectral redundancy.

There is a trade-off when selecting the modulation format for each of the subcarriers. QAM has a better BER than PAM for the same modulation order under stationary white noise [1]; however, PAM includes conjugate spectral redundancy which can be incorporated into the PFD for a signal processing gain over using QAM. The selection of the modulation format for each of the subcarriers is dependent on the requirements for the communication link and the characteristics of the received signal including the type and parameters of the interference and the SNR. The optimization in Section 4.5 provides one set of results where these trade-offs are incorporated by minimizing the BER while maximizing the spectral redundancy.

\subsection{Interference model}

A single carrier 16-QAM signal is used as interference to show the ability of the PFD to mitigate wideband heterogeneous interference. The analytic representation for the interference is [1]:

$$
i(t)=\sum_{k=-\infty}^{+\infty} s_{k} p(t-k T) e^{j 2 \pi f_{0} t},
$$

where $s_{k}$ is the $k^{\text {th }} 16$-QAM symbol and $p(t)$ is a square root raised cosine (SRRC) pulse-shaping filter with roll-off factor 0.35 and $f_{0}$ is the center frequency of the signal. Specific parameters for the interference such as the power and bandwidth are specified in Section 4.1.

The cycle frequencies of a 16-QAM signal are [26]:

$$
\alpha=\frac{k}{T}, k=0, \pm 1, \pm 2, \ldots
$$

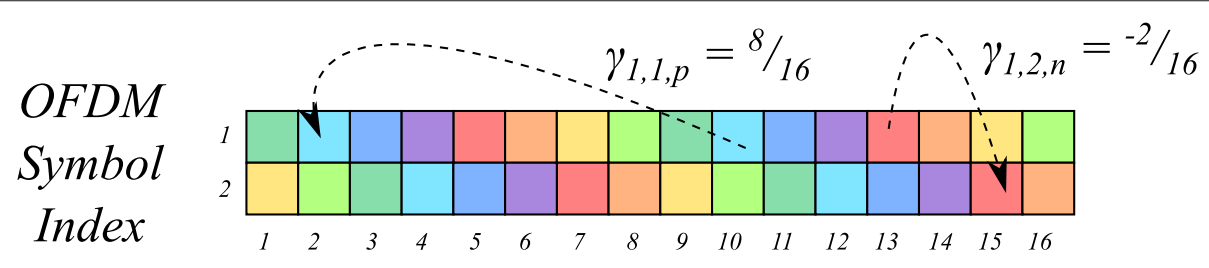

Subcarrier Index

Fig. 2 Spectral redundancy is distributed across both time and frequency within this pattern of $B=2$ OFDM symbols, $N=16$ subcarriers, and $M=8$ data-symbols. Three CFs illustrate the time-varying spectral redundancy. The arrows indicate in which direction the FS is performed 


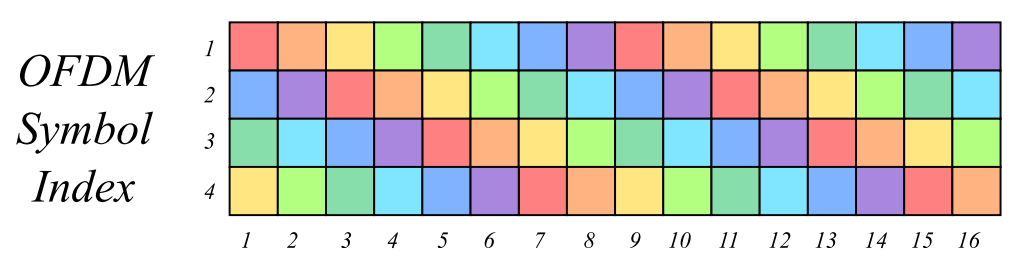

Subcarrier Index

Fig. 3 An example of a repetition pattern for $B=4$ OFDM symbols, $N=16$ subcarriers, and $M=8$ data-symbols

The SRRC pulse shaping reduces the energy of the spectral copies beyond the symbol rate; therefore, for practicality, the cycle frequencies are limited to:

$$
\alpha=\left\{0, \frac{1}{T},-\frac{1}{T}\right\}
$$

\subsection{Paramorphic FRESH demodulator}

The analytic representation of the PFD is given in this section. The PFD structure only requires knowledge of the cycle frequencies within the received signal as in (31) and (32). The cycle frequencies $\alpha_{c, b, u}$ and $\beta_{c, b, u}$ determine how many filtering branches are located within the PFD. The cycle frequencies of the PMW can be determined from Section 3.2 and the cycle frequencies of the interference from Section 3.3. The additional knowledge required for MMSE filter weights is described in Section 3.5.

The fast Fourier transform (FFT) is used to demodulate the OFDM signal, transforming the time domain signal into frequency domain symbols. A novel FRESH filter is employed in the frequency domain to exploit the timevarying spectral redundancy of the received signal. Each block of $B$ OFDM symbols are buffered in memory using a series of delay lines which allows them to be jointly filtered. The estimate $\hat{D}_{l, c}$ of the $c^{\text {th }}$ symbol of the $l^{\text {th }}$ block $D_{l, c}$ is given in (30), and the filtering structure is illustrated in Figs. 4 and 5 [18],

$$
\begin{aligned}
\hat{D}_{l, c}(f)= & \sum_{b=0}^{B-1}\left(\sum_{u=0}^{U_{c, b}-1} G_{c, b, u}(f) X_{l, b}\left(f-\alpha_{c, b, u}\right)\right. \\
& \left.+\sum_{v=0}^{V_{c, b}-1} H_{c, b, v}(f) X_{l, b}^{*}\left(-f+\beta_{c, b, v}\right)\right) .
\end{aligned}
$$

$U_{c, b}$ is the number of FSs in the FRESH filter between OFDM symbol $c$ and OFDM symbol $b$. Similarly, $V_{c, b}$ is the number of FSs in the FRESH filter corresponding to the conjugate spectrally redundant symbols in OFDM symbol $c$ and OFDM symbol $b$.

The estimate of the PMW (30) is a function of the FSs $\left\{\alpha_{c, b, u}\right\}$ and $\left\{\beta_{c, b, v}\right\}$ in the FRESH filter. The set of CFs of the spectral correlation for the PMW, $\left\{\gamma_{d, c, b, u}\right\}$, and interference, $\left\{\gamma_{i, c, b, u}\right\}$, as well as their differences are included in the set of FSs $\left\{\alpha_{c, b, u}\right\}$ as in (8):

$$
\begin{aligned}
\left\{\alpha_{c, b, u}\right\}=\{ & \gamma_{d, c, b, 0}, \gamma_{d, c, b, 1}, \gamma_{d, c, b, 2}, \ldots, \\
& \gamma_{i, c, b, 0}, \gamma_{i, c, b, 1}, \gamma_{i, c, b, 2}, \ldots, \\
& \gamma_{d, c, b, 0}-\gamma_{i, c, b, 0},-\gamma_{d, c, b, 0}+\gamma_{i, c, b, 0}, \\
& \gamma_{d, c, b, 0}-\gamma_{i, c, b, 1},-\gamma_{d, c, b, 0}+\gamma_{i, c, b, 1}, \\
& \ldots . \\
& \gamma_{d, c, b, 1}-\gamma_{i, c, b, 0},-\gamma_{d, c, b, 1}+\gamma_{i, c, b, 0}, \\
& \gamma_{d, c, b, 1}-\gamma_{i, c, b, 1},-\gamma_{d, c, b, 1}+\gamma_{i, c, b, 1}, \\
& \ldots\} .
\end{aligned}
$$

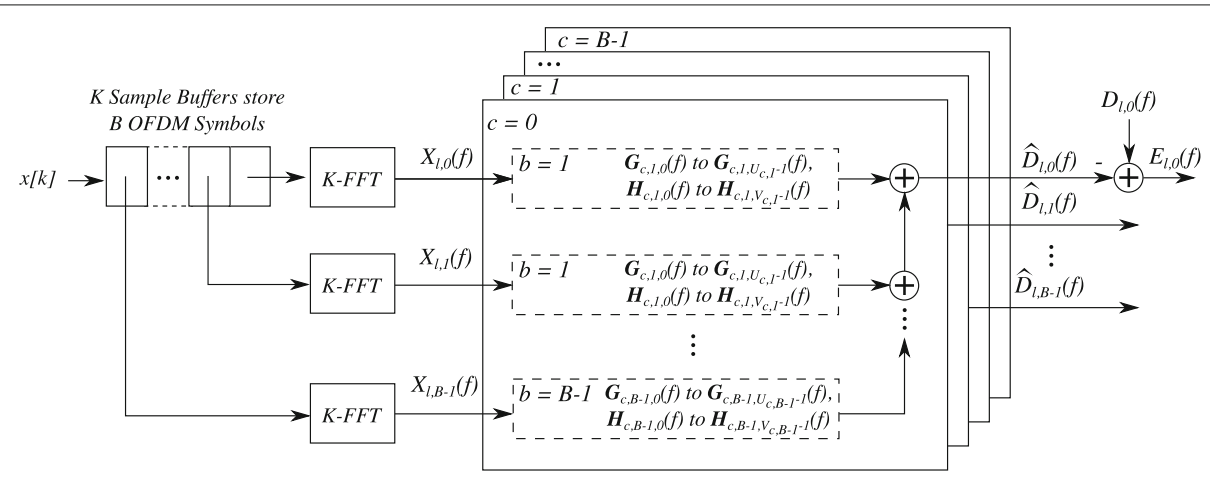

Fig. 4 The PFD structure that exploits spectral redundancy across time and frequency by demodulating and applying a novel FRESH filter. The depth in the image indicates a similar filtering structure with different filter weights for each of the sets of OFDM symbols 


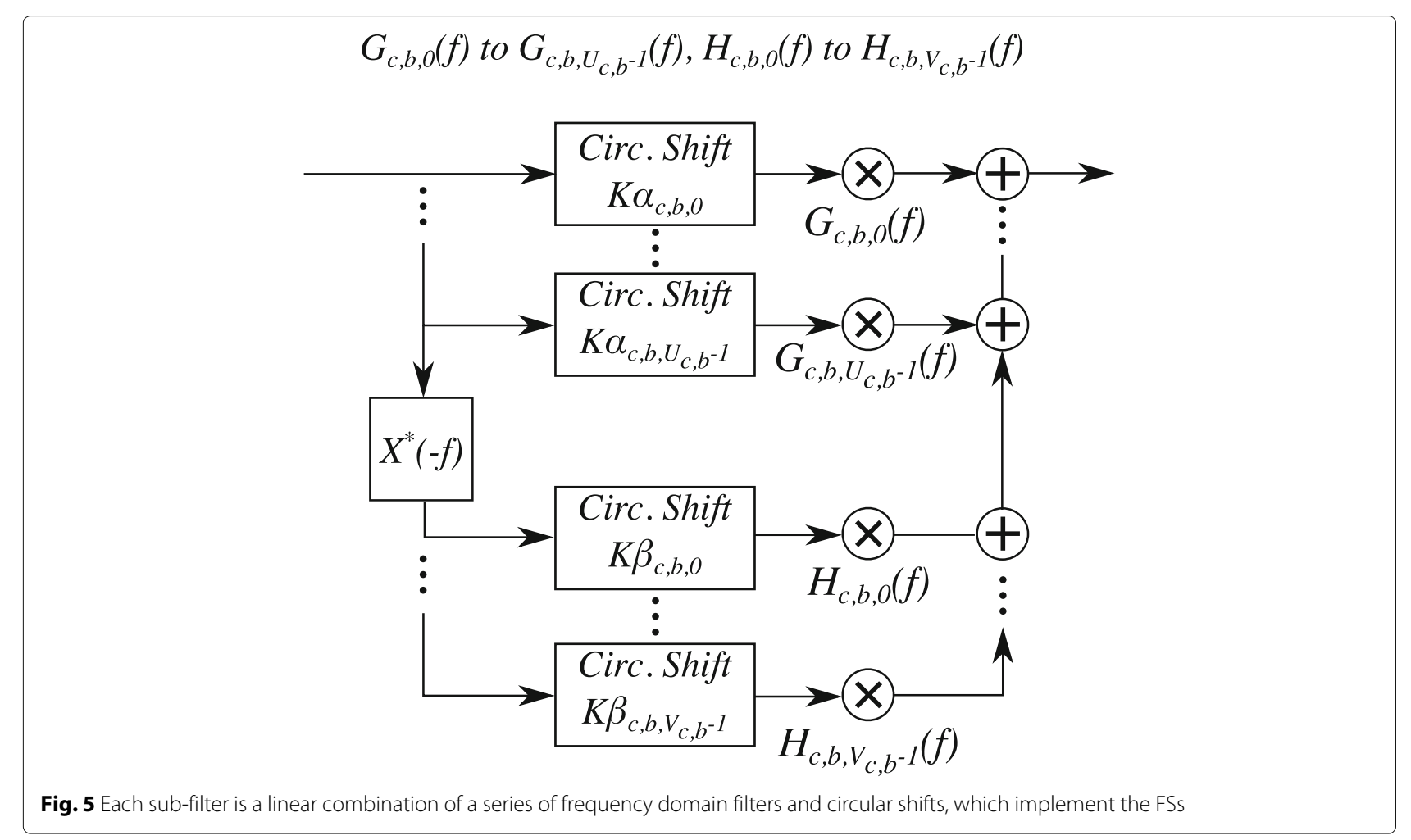

The set of FSs $\left\{\beta_{c, b, v}\right\}$ is formed using the set of CFs of the conjugate spectral correlation for the PMW, $\left\{\zeta_{d, c, b, u}\right\}$, and interference, $\left\{\zeta_{i, c, b, v}\right\}$, and their differences:

$$
\begin{aligned}
\left\{\beta_{c, b, v}\right\}= & \left\{\zeta_{d, c, b, 0}, \zeta_{d, c, b, 1}, \zeta_{d, c, b, 2}, \ldots,\right. \\
& \zeta_{i, c, b, 0}, \zeta_{i, c, b, 1}, \zeta_{i, c, b, 2}, \ldots, \\
& \zeta_{d, c, b, 0}-\zeta_{i, c, b, 0},-\zeta_{d, c, b, 0}+\zeta_{i, c, b, 0}, \\
& \zeta_{d, c, b, 0}-\zeta_{i, c, b, 1},-\zeta_{d, c, b, 0}+\zeta_{i, c, b, 1}, \\
& \ldots, \\
& \zeta_{d, c, b, 1}-\zeta_{i, c, b, 0},-\zeta_{d, c, b, 1}+\zeta_{i, c, b, 0}, \\
& \zeta_{d, c, b, 1}-\zeta_{i, c, b, 1},-\zeta_{d, c, b, 1}+\zeta_{i, c, b, 1}, \\
& \ldots\} .
\end{aligned}
$$

It is assumed that the cycle frequencies, and thus the signaling rate, of the interference is known. This information can be arrived at through spectrum sensing, or if the interference is a legacy user in a shared spectrum band it will already be known. Mismatches or errors in the estimated cycle frequencies and the true cycle frequencies due to real world impairments can be corrected through adaptive algorithms [27].

Spectrally redundant information which does not vary with time, either present in the PMW or the interference, can be included in (31) and (32) by setting the FSs appropriately. Consider the example of a single-carrier QPSK interferer with symbol period $T$, whose CFs are $\left\{0, \pm \frac{1}{T}, \pm \frac{2}{T}, \ldots\right\}[26]$. The CFs do not vary with time, thus $\gamma_{i, c, b, u}=\varnothing$ when $c \neq b$, and $\gamma_{i, c, b, 0}=0, \gamma_{i, c, b, 1}=$ $\frac{1}{T}, \gamma_{i, c, b, 2}=-\frac{1}{T}, \ldots$, for $c=b$.

\subsection{MMSE filter weights}

The MMSE filter weights for $G_{c, b, u}(f)$ and $H_{c, b, u}(f)$ of the PFD from (30) are derived within this section. The MMSE filter weights are optimal as they require knowledge of the desired signal, a common assumption used in the Wiener filter [3] derivation and previous MMSE FRESH filter weight derivations [2, 28, 29]. The MMSE filter weights are derived to find an upper bound on the performance of the filtering which future work can use as a reference. LMS algorithms for FRESH filters such as $[10,11]$ can be incorporated to adapt the filter weights using preambles for training or blind adaptation [30], such as decision-directed training or property restoration, and whose performance can be measured against the MMSE filter weights derived here.

The derivation of the MMSE filter weights [18] for $G_{c, b, u}(f)$ and $H_{c, b, v}(f)$ of the paramorphic FRESH demodulator proceeds in the same fashion as the derivation for the time-domain FRESH filter from Section 2.3. The filter error is established in (33) and the MSE in (34):

$$
\begin{aligned}
& E_{l, c}(f)=D_{l, c}(f)-\hat{D}_{l, c}(f), \\
& E_{M S E}(f)=\mathbb{E}\left\{E_{l, c}(f) E_{l, c}^{*}(f)\right\} .
\end{aligned}
$$


The MSE is minimized by taking its derivative with respect to both $G_{c, b, u}(f)$ and $H_{c, b, v}(f)$ and setting equal to zero in (35) and (36):

$$
\begin{gathered}
\frac{\partial E_{\mathrm{MSE}}(f)}{\partial G_{c, p, k}^{*}(f)}=0, \\
\frac{\partial E_{\mathrm{MSE}}(f)}{\partial H_{c, m, n}^{*}(f)}=0 .
\end{gathered}
$$

Solving for the derivatives results in two orthogonal projections, (37) and (38):

$$
\begin{aligned}
& \mathbb{E}\left\{E_{l, c}(f) X_{l, p}^{*}\left(f-\alpha_{c, p, k}\right)\right\}=0, \\
& \mathbb{E}\left\{E_{l, c}(f) X_{l, m}\left(-f+\beta_{c, m, n}\right)\right\}=0 .
\end{aligned}
$$

The first projection states that the filter error of the $c^{\text {th }}$ OFDM symbol is orthogonal to the conjugate of the received $p^{\text {th }}$ OFDM symbol when frequency-shifted by $\alpha_{c, p, k}$.

The frequency domain error (33) is then substituted into the projections (37) and (38) to produce the optimal filter design Eqs. (41) and (42). The design equations include new ways of representing the spectral correlation. For example, $S_{d_{c, x, p}}^{\alpha_{c, p}}\left(f-\frac{\alpha_{c, p, k}}{2}\right)$ is the cross spectral correlation of the $c^{\text {th }}$ desired OFDM symbol from each block with the $p^{\text {th }}$ received OFDM symbol from each block at FS $\alpha_{c, p, k}$, represented by:

$$
\begin{aligned}
S_{d_{c}, x_{p}}^{\alpha_{c, p, k}} & \left(f-\frac{\alpha_{c, p, k}}{2}\right)=\lim _{L \rightarrow \infty} \frac{1}{L} \\
& \cdot \sum_{l_{1}, l_{2}=0}^{L-1} D_{l_{1}, c}(f) X_{l_{2}, p}^{*}\left(f-\alpha_{c, p, k}\right) .
\end{aligned}
$$

\subsection{Theoretical SINR}

In order to validate the simulation results, the theoretical signal to interference and noise ratio (SINR) at the output of the PFD is derived. The theoretical SINR (40) for the $c^{\text {th }}$ OFDM symbol is:

$$
\lambda_{l}=\frac{\mathbb{E}\left\{\left|D_{l, c}(f)\right|^{2}\right\}}{\mathbb{E}\left\{\left|D_{l, c}(f)-\hat{D}_{l, c}(f)\right|^{2}\right\}},
$$

where $D(f)$ is from (22) and $\hat{D}_{l, c}(f)$ is from (30). The denominator is the MSE and is expanded in (46), with additional terms given in (47) and (48), and where $\Re$ is the real operator. The analytical SINR from (40) is used to validate the simulation results for the PFD in Section 4.

$$
\begin{aligned}
S_{d_{c, x_{p}}}^{\alpha_{c, p, k}}\left(f-\frac{\alpha_{c, p, k}}{2}\right)= & \sum_{b=0}^{B-1}\left(\sum_{u=0}^{U_{c, b}-1} G_{c, b, u}(f) S_{x_{c, x_{b}}}^{\alpha_{c, p, k}-\alpha_{c, b, u}}\left(f-\frac{\alpha_{c, p, k}+\alpha_{c, b, u}}{2}\right)\right. \\
& \left.+\sum_{v=0}^{V_{c, b}-1} H_{c, b, v}(f) S_{x_{c}, x_{b}^{*}}^{\beta_{c, b}-\alpha_{c, p, k}}\left(f-\frac{\beta_{c, b, v}+\alpha_{c, p, k}}{2}\right)^{*}\right)
\end{aligned}
$$

$$
p=0,1, \ldots, B-1 ; \quad k=0,1, \ldots, U_{c, p}-1
$$

$$
\begin{aligned}
S_{d_{c}, x_{m}^{n}}^{\beta_{c, m},}\left(f-\frac{\beta_{c, m, n}}{2}\right)= & \sum_{b=0}^{B-1}\left(\sum_{u=0}^{U_{c, b}-1} G_{c, b, u}(f) S_{x_{c}, x_{b}^{n}}^{\beta_{c, m, n}-\alpha_{c, b, u}}+\left(f-\frac{\beta_{c, m, n}+\alpha_{c, b, u}}{2}\right)\right. \\
& \left.\sum_{v=0}^{V_{c, b}-1} H_{c, b, v}(f) S_{x_{c}, x_{b}}^{\beta_{c, m, n}-\beta_{c, b, v}}+\left(-f+\frac{\beta_{c, m, n}+\beta_{c, b, v}}{2}\right)\right)
\end{aligned}
$$

$$
m=0,1, \ldots, B-1 ; \quad n=0,1, \ldots, V_{c, m}-1
$$

\subsection{Joint optimization of waveform and demodulator}

Cognitive radios most commonly adapt waveform parameters such as transmit power, modulation type and order, and code rate and type to satisfy objectives of BER, throughput, and spectral efficiency [23,31]. The ability to design the waveform for interference mitigation using symbol repetition allows for another degree of freedom within the optimization.

The fitness function is used to combine the performance of several objectives into a single number where a larger fitness function directly translates to a more suitable solution to the optimization. In this approach, a linear fitness function (43),

$$
\phi=w_{1} \phi_{1}+w_{2} \phi_{2},
$$

combines the objective of the BER, $\phi_{1}$, and the objective of the spectral efficiency, $\phi_{2}$. The weights $w_{1}$ and $w_{2}$ are set such that $w_{1}+w_{2}=1$. The objectives $\phi, \phi_{1}$, and $\phi_{2}$ are all bounded by $[0,1]$. The objective functions for the BER and spectral efficiency are given by (44) and (45) [32]:

$$
\begin{aligned}
& \phi_{1}=\frac{\log _{10}(0.5)-\log _{10}\left(\mathrm{BER}_{M}\right)}{\log _{10}(0.5)-\log _{10}\left(\mathrm{BER}_{T}\right)}, \\
& \phi_{2}=\frac{\eta}{\eta_{\max }} .
\end{aligned}
$$

$\mathrm{BER}_{T}$ is a target $\mathrm{BER}$, and $\mathrm{BER}_{M}$ is the measured BER. The target BER is a minimum BER threshold, where if the threshold is not met, the solution is penalized by reducing the fitness of the solution. The measured BER is the actual BER of the PMW, requiring knowledge of the desired signal, which is compared against the BER 
threshold when computing the fitness function. Knowledge of the desired signal is also needed in order to form the MMSE filter weights of the PFD. In practical systems, training sequences or pilot sequences are transmitted to provide a known signal. The average information bits per symbol, $\eta$, comes from the modulation and incorporates the reduction in spectral efficiency due to coding as well as symbol repetition. The weights are set $w_{1}=\frac{2}{3}$ and $w_{2}=\frac{1}{3}$ to ensure the optimization does not select a solution which produces a poor BER but has an abnormally high spectral efficiency.

$$
\begin{aligned}
\mathbb{E}\left\{\left|D_{l, c}-\hat{D}_{l, c}\right|^{2}\right\}=S_{d}(f)- & 2 \Re\left(\mathbb{E}\left\{D_{l, c}(f) \hat{D}_{l, c}^{*}(f)\right\}\right) \\
+ & \mathbb{E}\left\{\hat{D}_{l, c}(f) \hat{D}_{l, c}^{*}(f)\right\}
\end{aligned}
$$

$$
\begin{aligned}
\mathbb{E}\left\{D_{l, c}(f) \hat{D}_{l, c}^{*}(f)\right\}= & \sum_{b=0}^{B-1}\left(\sum_{u=0}^{U_{c, b}-1} G_{c, b, u}^{*}(f) S_{d_{c}, x_{b}}^{\alpha_{c, u}}\left(f-\frac{\alpha_{c, b, u}}{2}\right)\right. \\
& \left.+\sum_{v=0}^{V_{c, b}-1} H_{c, b, v}^{*}(f) S_{d_{c, x}, x_{b}^{*}}^{\beta_{c, b v}}\left(f-\frac{\beta_{c, b, v}}{2}\right)\right)
\end{aligned}
$$

$$
\begin{aligned}
& \mathbb{E}\left\{\hat{D}_{l, c}(f) \hat{D}_{l, c}^{*}(f)\right\}=\sum_{b=0}^{B-1} \sum_{z=0}^{B-1} \sum_{u=0}^{U_{c, b}-1} \sum_{m=0}^{U_{c, b}-1} G_{c, b, u}(f) G_{c, z, m}^{*}(f) . \\
& S_{x_{b}, x_{z}}^{\alpha_{c, z, m}-\alpha_{c, b, u}}\left(f-\frac{\alpha_{c, b, u}+\alpha_{c, z, m}}{2}\right) \\
& +2 \Re\left\{\sum_{b=0}^{B-1 B-1} \sum_{z=0}^{B-1} \sum_{u=0}^{U_{c, b}-1} \sum_{n=0}^{1 V_{c, b}-1} G_{c, b, u}(f) H_{c, z, n}^{*}(f)\right. \\
& \left.S_{x_{b}, x_{z}}^{\beta_{c, n, n}-\alpha_{c, b, u}}\left(f-\frac{\beta_{c, b, n}+\alpha_{c, b, u}}{2}\right)\right\} \\
& +\sum_{b=0}^{B-1} \sum_{z=0}^{B-1} \sum_{v=0}^{V_{c, b}-1} \sum_{n=0}^{V_{c, b}-1} H_{c, b, v}(f) H_{c, z, n}^{*}(f) \\
& s_{x_{b}, x_{z}}^{\beta_{c, n}-\beta_{c, b, v}}\left(-f+\frac{\beta_{c, b, v}+\beta_{c, z, n}}{2}\right)
\end{aligned}
$$

\section{Simulation results}

Simulation results are used to show the ability of the PMW and PFD to mitigate interference and make comparisons to traditional filtering techniques and ECC. The simulated scenario is motivated by the co-existence of $5 \mathrm{G}$ cellular systems and satellite communications within the same band [33]. The SINR is used to compare against filtering techniques in Section 4.2, and the BER is used to compare against ECCs in Section 4.3. Simulated annealing is used in Section 4.5 to optimize the BER and spectral efficiency when under interference. A detailed complexity analysis of the PFD is given in Section 4.6.

\subsection{Simulation parameters}

In Sections 4.2 and 4.3, five different techniques are compared for their ability to mitigate interference. The techniques are Wiener filtering, maximal ratio combining (MRC), the proposed PFD, soft decision convolutional codes, and soft decision LDPC codes. The spectral redundancy of the interferer is exploited within the PFD results, but for no other methods. The LDPC codes used are those defined in the digital video broadcasting second generation (DVB-S2) standard [34]. The SINR and BER are computed within the same set of Monte Carlo simulations. The length of the input signal to the FRESH filter is a minimum of $10^{5}$ samples for each run of the simulation, and the simulations continue until a minimum of $1000 \mathrm{bit}$ errors are observed.

The 16-QAM interference model from Section 3.3 is used in all of the following simulations, although the power and bandwidth are varied. The interference has high SIR values within the passband of the desired signal, and examples of the received spectrum under these conditions are given in Fig. 6a, b.

\subsection{SINR performance}

The SINR after the Wiener filter, MRC, and the PFD are all compared using the simulation parameters referenced in Section 4.1. The SINR is simulated for a series of $E_{b} / N_{0}$ values where the interference to noise ratio is fixed at $P_{i} / N_{0}=20 \mathrm{~dB}$, and the interference bandwidth covers $1 / 2$ and $1 / 4$ of that of the OFDM signal. The energy per bit $E_{b} / N_{0}$ is in relation to information bits, with QPSK being used on each of the 64 subcarriers of the OFDM signal.

The SINR is plotted in Fig. 7a, b, and it can be seen that significant gains can be achieved by using the PFD over MRC and the Wiener filter. The gains over the Wiener filter are about 6 and $4 \mathrm{~dB}$ at $E_{b} / N_{0}=10 \mathrm{~dB}$ when the interference covers $1 / 2$ and $1 / 4$ the PMW bandwidth. The gains over MRC are about 1.5 and $1 \mathrm{~dB}$ when the interference covers $1 / 2$ and $1 / 4$ the PMW bandwidth.

To cancel $K$ cyclostationary, interferers in the absence of noise each symbol must be repeated $K+1$ times [2]; thus, for the single interferer case, each symbol only needs to be repeated twice. Repeating each symbol four times or more will improve the estimate of the received signal as it will still mitigate the stationary white noise but it is subject to diminishing returns. Increasing from $1 / 2$ rate symbol repetition to $1 / 4$ rate produces only a small improvement in SINR. 

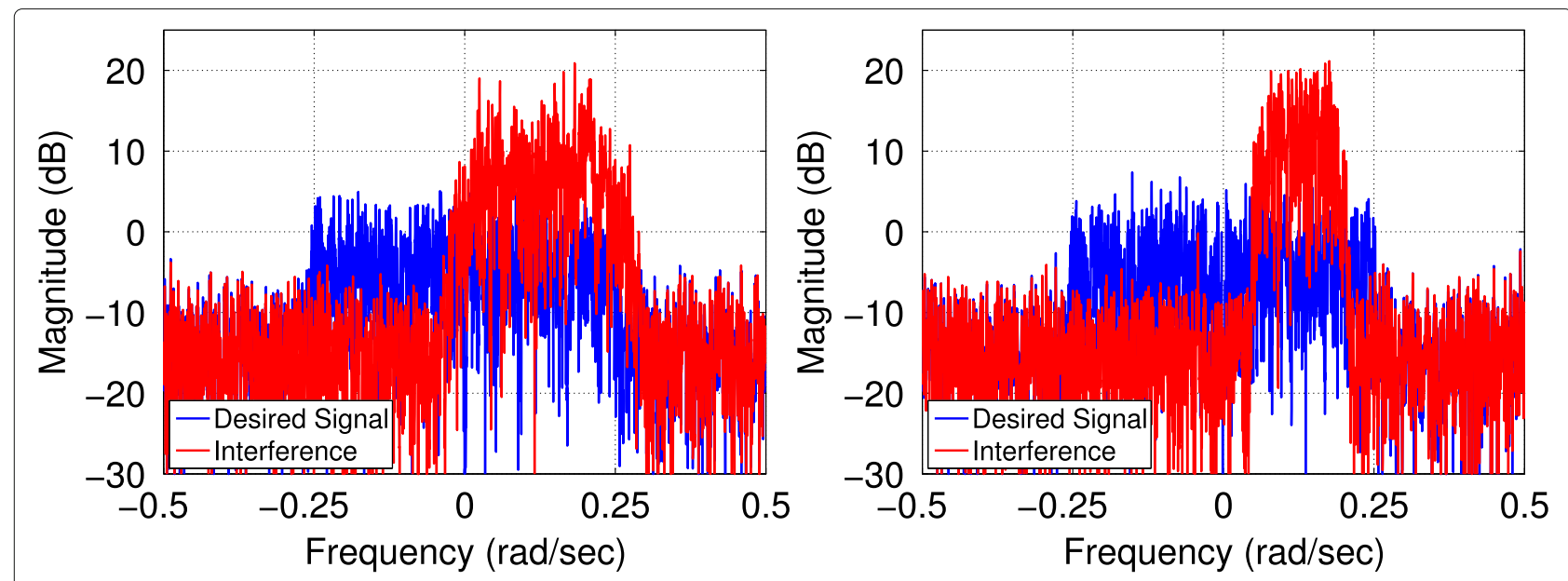

Fig. 6 Two plots showing the spectrum of the received signal when $E_{b} / N_{0}=7 \mathrm{~dB}$ and $P_{i} / N_{0}=20 \mathrm{~dB}$. The blue line represents the desired signal, and the red line represents the interference. a An example of the spectrum when theinterference covers $1 / 2$ of the bandwidth. $\mathbf{b}$ An example of the spectrum when the interference covers $1 / 4$ of the bandwidth

\subsection{BER versus desired signal $E_{b} / N_{0}$}

The results of the BER being simulated for the Wiener filter, PFD, convolutional coding, and LDPC are given in Fig. 8a, b using the simulation parameters and interference model of Section 4.1. For these results, the PMW uses QPSK on each of its 64 subcarriers, the interference power is $P_{i} / N_{0}=20 \mathrm{~dB}$ and the bandwidth of the interference is $1 / 2$ and $1 / 4$ that of the PMW in Fig. $8 \mathrm{a}, \mathrm{b}$, respectively.

The PFD outperforms both the Wiener filter, the LDPC, and the convolutional codes in these two scenarios due to its optimal combining of spectral redundancy, even with only $1 / 2$ rate symbol repetition. The PFD can improve the BER further by using $1 / 4$ rate symbol repetition but with diminishing returns. Since the received signal is dominated by cyclostationary interference and not stationary noise, assumptions built into LDPC and convolutional codes break down and thus produce BERs of worse than $10^{-1}$ for the considered values of $E_{b} / N_{0}$. However, using a hybrid approach of the PFD and an ECC can improve the BER more than either method alone, by reducing the BER by $1000 x$ in comparison to the next best method at high $E_{b} / N_{0}$ values.
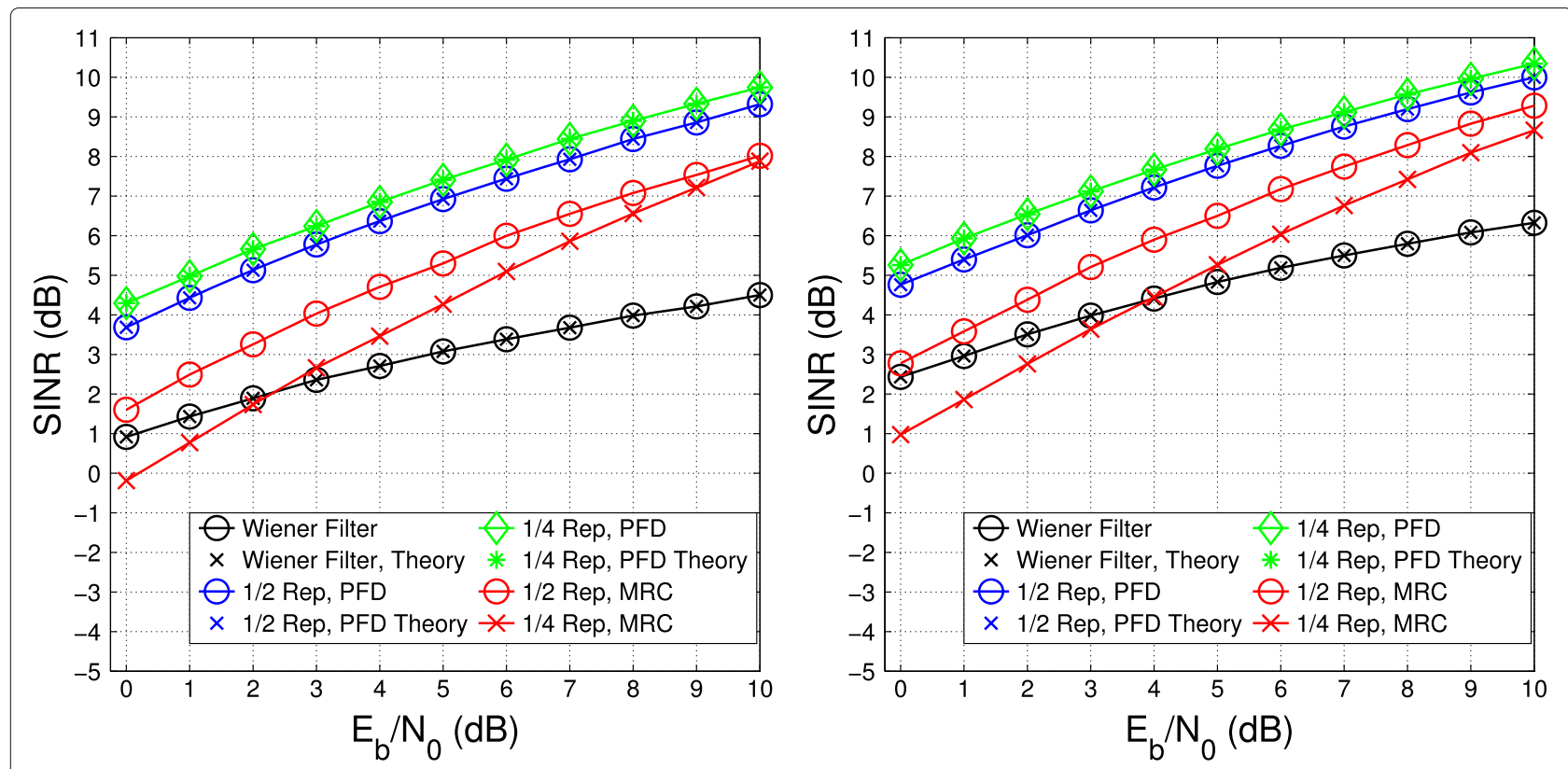

Fig. 7 The SINR plots comparing the performance of the Wiener filter (black), 1/2 Rate Repetition PFD (blue), 1/4 Rate Repetition PFD (green), and MRC (red). The abbreviation Rep. refers to symbol repetition. a Bandwidth overlap of 1/2. b Bandwidth overlap of 1/4 

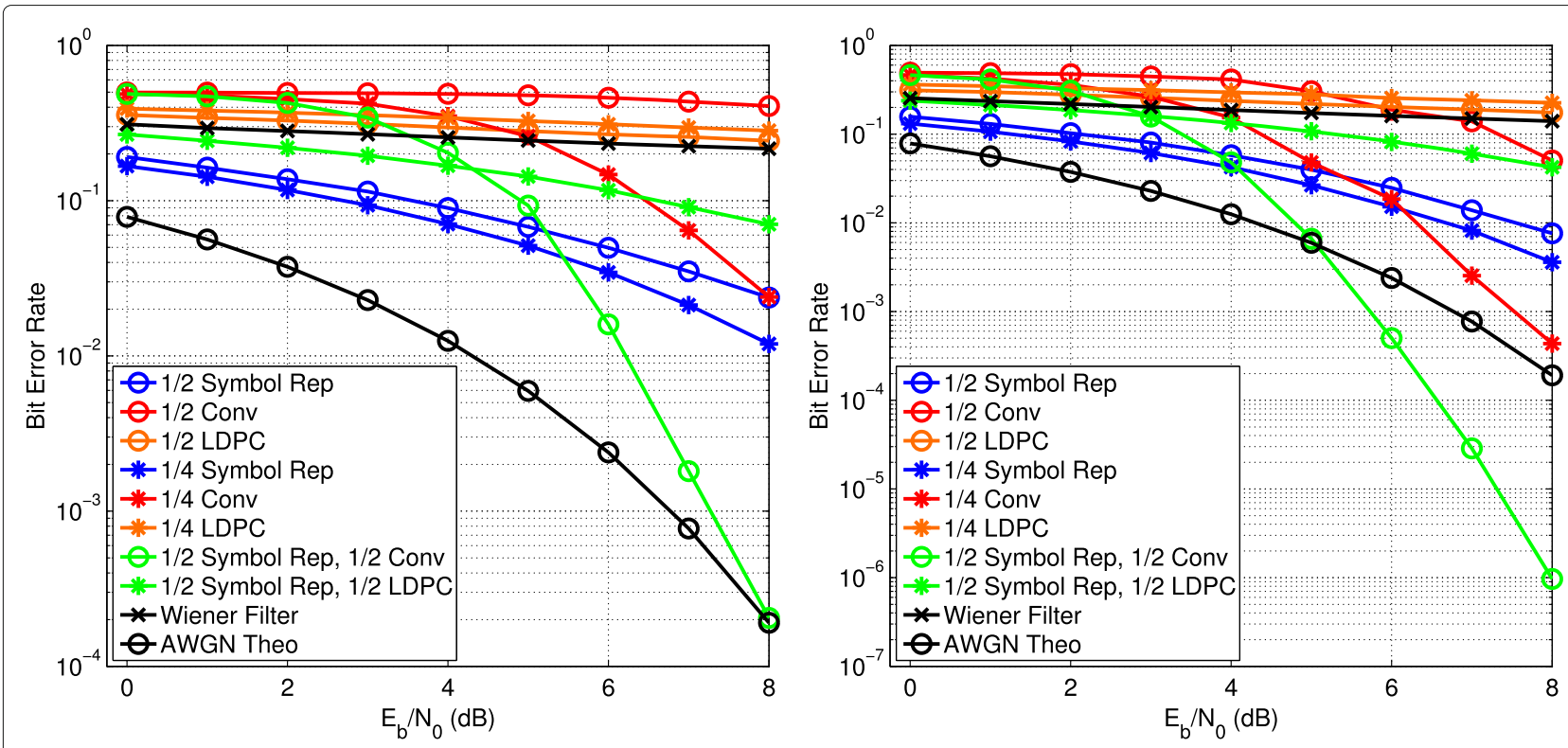

Fig. 8 The BER curves comparing the performance of the 1/2 Rate Symbol Repetition PFD (blue circle), 1/4 Rate Symbol Repetition PFD (blue star), 1/2 Rate Convolutional Code (red circle), 1/4 Rate Convolutional Code (red star), 1/2 Rate LDPC (orange circle), 1/4 Rate LDPC (orange star), combined 1/2 Rate Symbol Repetition and 1/2 Rate Convolutional Code (green circle), combined 1/2 Rate Symbol Repetition and 1/2 Rate LDPC (green star), Wiener Filter (black x). The AWGN Theoretical is plotted with a black circle. All BER curves are plotted as a function of $E_{b} / N_{0}$. a Bandwidth overlap of 1/2. b Bandwidth overlap of 1/4

With the hybrid approach, the PFD is able to mitigate a large portion of the CS interference allowing the convolutional code to operate on the resulting signal which is mostly corrupted by stationary noise. It is for this reason that the hybrid approach exceeds the BER for the theoretical AWGN curve in Fig. 8b. Enough CS interference is mitigated by the PFD that the convolutional code can then correct residual bit errors caused by the stationary noise.

The received signal is dominated by cyclostationary interference, and the time-varying nature of the PFD transforms the stationary white noise into time-varying noise. Additionally, the frequency shifts within the PFD color the white noise within the signal. The resulting noise at the output of the PFD is now non-stationary, periodically time-varying, and colored, and it will include any remaining cyclostationary interference that was unable to be removed by the PFD. All of these qualities severely violate the assumptions of stationary white Gaussian noise built into the standard BER equations, which accounts for the distance between the theoretical BER curves and the Monte Carlo results. The violation of the assumptions also prevent an approximation of the theoretical BER by using the SINR results obtained in Section 4.2.

The expected reason for the bad performance of LDPC codes comes from the shape of the BER curve, or the thresholding effect. While above this threshold, LDPC codes perform well in AWGN, but below it, the codes provide no error correction capability. In [35], it is shown that cyclostationary noise can have a dramatic effect on the performance of LDPC codes at much smaller interference powers than what has been simulated in this paper.

Figure $9 \mathrm{a}, \mathrm{b}$ shows the BER when under frequencyselective fading; the magnitude of the frequency response is given in Fig. 10. The desired signal channel is $\Phi(z)=$ $(-0.35-1.06 j)+(-0.84+0.69 j) z^{-1}+(0.03-0.01 j) z^{-2}$ and the interference channel is $\Psi(z)=(-0.004+0.09 j)+$ $(-0.80+1.26 j) z^{-1}+(0.02-0.74 j) z^{-2}$. The delay $z^{-1}$ is defined according to the sampling rate. The simulated results use a normalized sampling frequency, with the ratio of the bandwidth of the PMW to the sampling ratio being $\frac{1}{2}$. The BER for the paramorphic approach actually improves relative to the case in which there is no fading. The FRESH filter can be thought of as an improved fractionally spaced equalizer [2], providing resistance to the fading effects. Additionally, the interference channel reduces the power of the interference on its left side, effectively reducing the bandwidth of the interference. Therefore, both BER curves are close to the case in which the bandwidth only covers $1 / 4$ of the bandwidth, Fig. $8 \mathrm{~b}$.

\subsection{BER versus interference power}

The impact of the interference power on the BER is given in Fig. 11a, b, as the bandwidth of the interference is $1 / 2$ and $1 / 4$ that of the desired signal, respectively. In these results, the power of the desired signal is held constant at $E_{b} / N_{0}=7 \mathrm{~dB}$ with QPSK being used on each of the 64 subcarriers and the interference power is varied. As the interference power increases, all approaches show a BER 

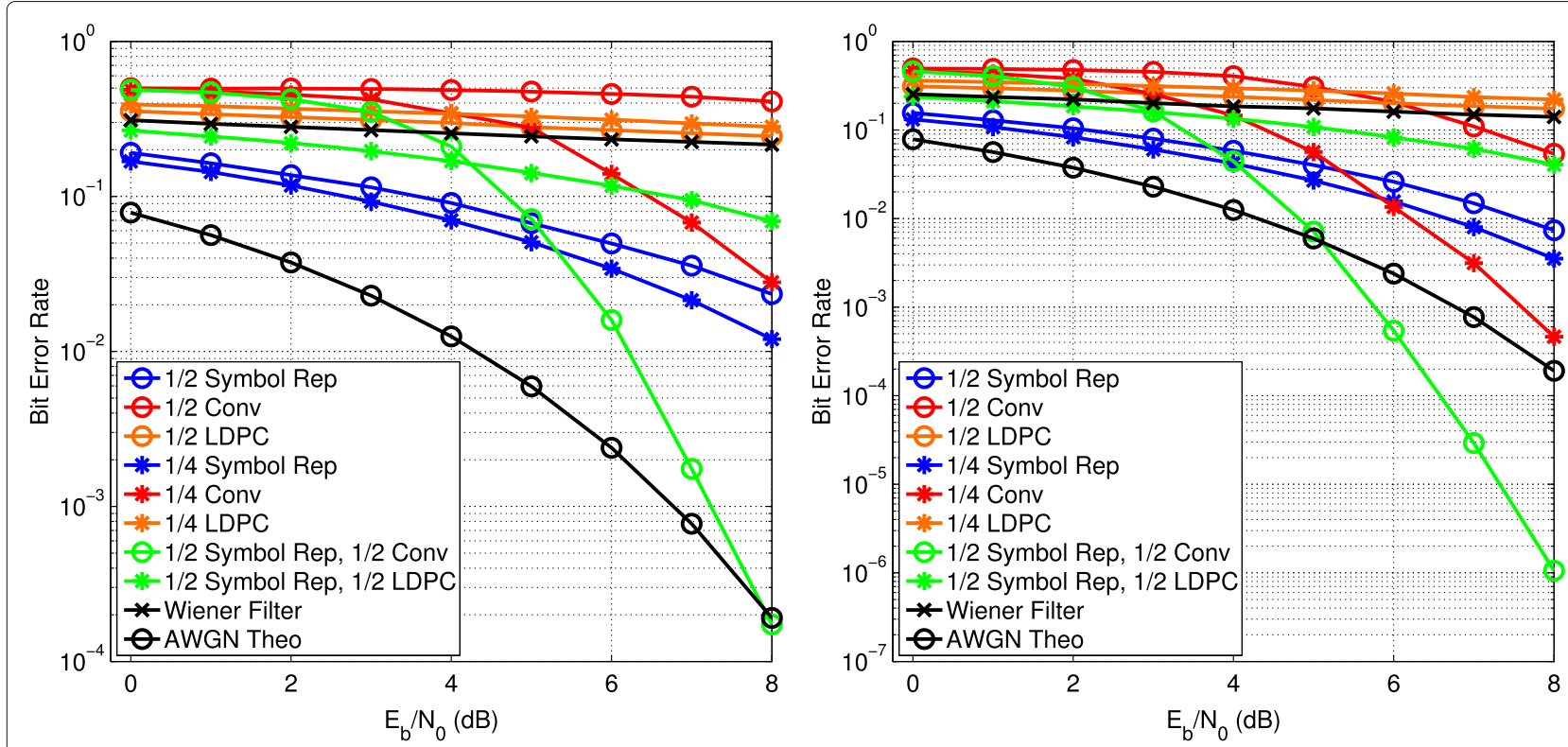

Fig. 9 The BER curves of the filtering methods and error correcting codes while under Rayleigh fading. The BER curves include simulated results for 1/2 Rate Symbol Repetition PFD (blue circle), 1/4 Rate Symbol Repetition PFD (blue star), 1/2 Rate Convolutional Code (red circle), 1/4 Rate Convolutional Code (red star), 1/2 Rate LDPC (orange circle), 1/4 Rate LDPC (orange star), combined 1/2 Rate Symbol Repetition and 1/2 Rate Convolutional Code (green circle), combined 1/2 Rate Symbol Repetition and 1/2 Rate LDPC (green star), Wiener Filter (black x). The AWGN Theoretical is plotted with a black circle. a Bandwidth overlap of 1/2. b Bandwidth overlap of 1/4

degradation but the PFD has the most graceful degradation, reducing the BER by less than an order of magnitude as the interference power is increased by $20 \mathrm{~dB}$.

The hybrid approach of using the PFD and a convolutional code works better than each approach alone for almost all interference power values. The PFD is resilient to the increases in interference power and mitigates enough of the CS interference that the convolutional code can be used to correct the bit errors caused by the residual noise. This results in the hybrid approach producing a better BER that also degrades slowly as a function of interference power.

The results demonstrate how an adaptive approach can be beneficial as the spectral environment changes. The PMW and PFD perform well in strong, wideband interference but do not perform as well as ECCs in purely AWGN

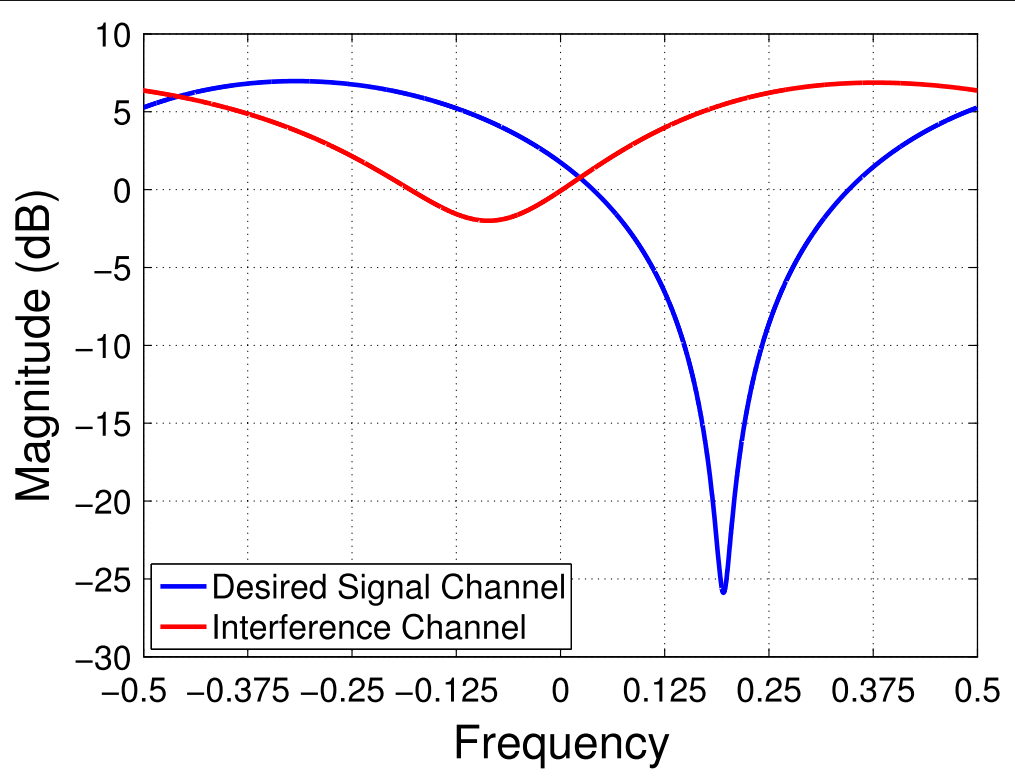

Fig. 10 The magnitude of the frequency response for the channel on the desired signal and interference 

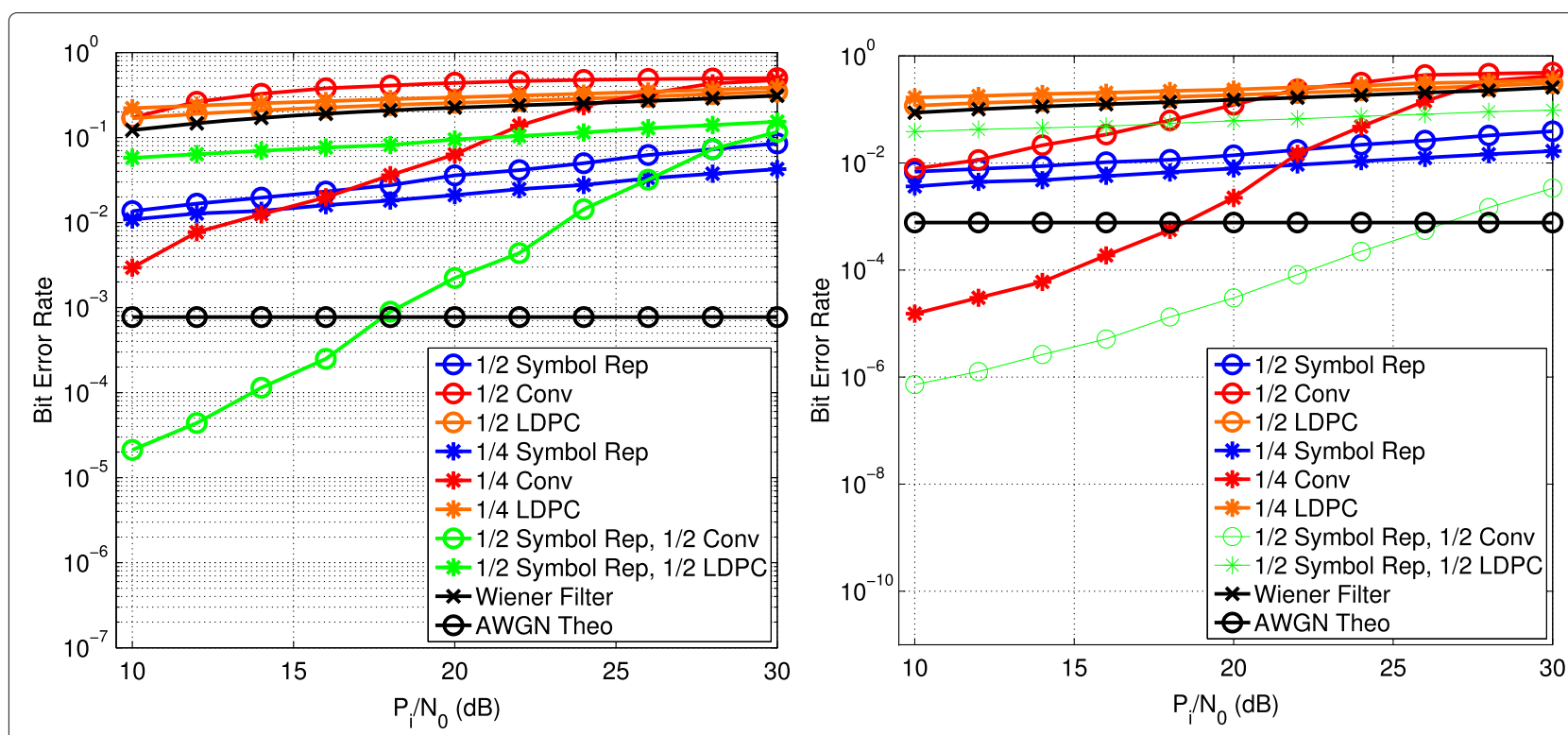

Fig. 11 The BER curves of the symbol repetition and filtering methods as a function of interference to noise power Pi/NO when Eb/NO=7 dB. The methods compared are 1/2 Rate Symbol Repetition PFD (blue circle), 1/4 Rate Symbol Repetition PFD (blue star), 1/2 Rate Convolutional Code (red circle), 1/4 Rate Convolutional Code (red star), 1/2 Rate LDPC (orange circle), 1/4 Rate LDPC (orange star), combined 1/2 Rate Symbol Repetition and 1/2 Rate Convolutional Code (green circle), combined 1/2 Rate Symbol Repetition and 1/2 Rate LDPC (green star), Wiener Filter (black x). The AWGN Theoretical is plotted with a black circle. a Bandwidth overlap of 1/2. b Bandwidth overlap of 1/4

channels. Balancing the redundancy used within ECCs and symbol repetition can therefore maximize the spectral efficiency while minimizing the BER as the spectral environment changes.

\subsection{Optimization results}

The optimization in Section 4.5 is used to maximize the spectral efficiency while minimizing the BER. The optimization of the PMW selects the symbol repetition rate, coding rate and modulation order for each of the 64 occupied subcarriers. The parameters of the 16-QAM interference described in Section 4.1 are used in this optimization, and it covers $1 / 2$ of the OFDM signal bandwidth. The power of both the desired signal and interference are both fixed for SIR $=-20 \mathrm{~dB}$, along with a fixed SNR $=10 \mathrm{~dB}$. This leaves the selection of the waveform parameters as the only variables, which will also set the $E_{b} / N_{0}$.

The optimization is designed to meet the target BERs $10^{-1}$ to $10^{-5}$ while maximizing the spectral efficiency of the waveform. Each optimization is run for 500 iterations of the simulated annealing algorithm, and each iteration is run until 10 bit errors are simulated. Due to computational and memory constraints within the simulation, 16 occupied subcarriers are used and spectral redundancy is only used across a single OFDM symbol, $B=1$. The amplitude of each subcarrier is selected to be within $(0,1]$, and Table 1 gives all options for the modulations, symbol repetition and convolutional code rates available for selection.

Figure 12 demonstrates how the simulated annealing algorithm balances the BER and the spectral efficiency to maximize the objective function. The initial iterations have the optimization looking for parameters which meet or exceed the target BER, and then, the spectral efficiency is improved through additional iterations. A record of the best set of outcomes is kept, which is represented by the blue line. The parameters which produced the maximum objective during the optimizations is given in Table 2 . Table 3 contains the results of the optimizations when the symbol repetition is disabled.

Tables 2 and 3 list the parameters and outcomes for the optimizations. Each row lists the target $\mathrm{BER}, \mathrm{BER}_{T}$, and the measured $\mathrm{BER}, \mathrm{BER}_{M}$. The symbol repetition rate and the code rate for the convolutional codes is also given.

Table 1 The list of options for the modulation, symbol repetition, and convolutional code rates from which the optimization can select

\begin{tabular}{ll}
\hline Modulations: & BPSK, QPSK, 8-PSK, 16-PSK, 16-QAM \\
\hline Symbol rep. rates: & none, $1 / 2,1 / 4,1 / 8,1 / 16$ \\
Conv. code rates: & none, $\frac{1}{1}, \frac{2}{3}, \frac{3}{4}, \frac{4}{5}, \frac{5}{6}, \frac{6}{7}, \frac{7}{8}, \frac{1}{2}, \frac{1}{3}, \frac{1}{4}, \frac{1}{5}, \frac{1}{6}, \frac{1}{7}, \frac{1}{8}, \frac{3}{5}, \frac{4}{7}, \frac{5}{9}, \frac{5}{8}, \frac{5}{7}, \frac{6}{11}, \frac{7}{13}, \frac{7}{12}, \frac{7}{11}, \frac{7}{10}, \frac{7}{9}$ \\
\hline
\end{tabular}



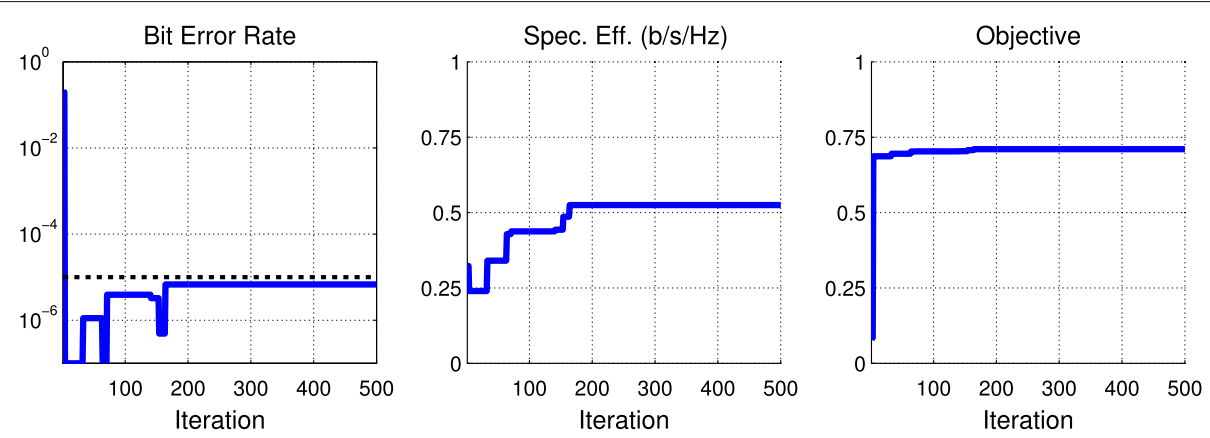

Fig. 12 The BER, spectral efficiency, and objective per iteration for the simulated annealing algorithm with a target BER of $10^{-4}$

The average number of information bits per data-symbol is given, which takes into account the repetition rate and the modulation order of the data symbols. The spectral efficiency includes the average number of information bits per data-symbol and the code rate.

It is seen in Fig. 8 a that using 1/2 rate symbol repetition provides the largest improvement in BER for the smallest amount of overhead, and a similar effect can be seen in Table 2. For the target BERs $10^{-1}$ to $10^{-4}$, the $1 / 2$ rate symbol repetition is used, while for the BER $10^{-5}$, the $1 / 4$ rate symbol repetition is needed to meet the BER.

When the PMW and PFD is not used, the BER approaches 0.5 for most target BERs as seen in Table 3 . As the BER cannot be controlled, the only way to maximize the objective function is therefore to maximize the spectral efficiency. The coding is able to meet the target BER for $10^{-1}$; however, its spectral efficiency is worse than using the PFD as it requires a $\frac{1}{8}$ code to do so. The PMW and PFD provide $16.5,12.3,11.2,11.0$, and $10.7 \mathrm{~dB}$ worth of SNR gain from BERs $10^{-5}$ to $10^{-1}$.

\subsection{Complexity analysis}

The improved performance of the PFD over traditional filtering methods comes at the cost of increased transform and filtering complexity, although clever design decisions can be made to minimize the complexity increases. A traditional FFT-based OFDM demodulator and one-tap equalizer is used as the baseline for comparison. The OFDM receiver discards the $N_{\mathrm{CP}}$ samples of the cyclic prefix, transforms the remaining signal using an $N$-point
FFT, and then applies $N$ complex weights to the frequency domain samples. The increases to the complexity of the PFD due additional discrete Fourier transforms (DFT) is presented in Section 4.6.1, and the increases in complexity within the filtering operation is given in Section 4.6.2.

\subsubsection{Complexity of additional DFTs}

Traditional OFDM receivers discard the cyclic prefix before taking the FFT over the OFDM symbol. This creates distortion when the received signal is affected by a large-power interferer because it generates phase discontinuities, resulting in additional distortion. The increased complexity comes from taking a DFT over the entire OFDM symbol, $N$ samples, along with the cyclic prefix $N_{\text {CP }}$ samples, which allows the phase to remain continuous. The additional complexity comes from this larger DFT, applying an IDFT to transform back to the time domain to discard the cyclic prefix after filtering, and a final FFT applied at the nominal size to obtain the frequency domain symbols.

The cyclic prefix length is often represented as a fraction of the number of samples within the body of the OFDM symbol, $N_{\mathrm{CP}}=N / 4$ for example. Efficiently computing the FFT requires a transform size that is a power of 2; however, the total input length of $N+N_{\mathrm{CP}}=5 \mathrm{~N} / 4$ is unlikely to be a power of 2 . In this case, another DFT implementation is needed.

It is more likely that the longer input sequence can be decomposed into two relative primes allowing use of the prime factorization algorithm (PFA) [36] to efficiently

Table 2 The results from the simulated annealing optimization using the PMW and PFD approach

\begin{tabular}{llllll}
\hline BERT & BER & Symbol rep. & Conv. code & Avg. info. bits & Spectral eff. \\
\hline $10^{-5}$ & $6.78 \times 10^{-6}$ & $1 / 4$ & $3 / 5$ & 0.88 & 0.53 \\
$10^{-4}$ & $8.86 \times 10^{-5}$ & $1 / 2$ & $3 / 5$ & 1.18 & 0.75 \\
$10^{-3}$ & $1.98 \times 10^{-4}$ & $1 / 2$ & $5 / 9$ & 1.50 & 0.83 \\
$10^{-2}$ & $5.21 \times 10^{-3}$ & $1 / 2$ & $3 / 5$ & 1.56 & 0.94 \\
$10^{-1}$ & $8.22 \times 10^{-2}$ & $1 / 2$ & None & 1.69 & 1.69 \\
\hline
\end{tabular}

Avg. Info. Bits is the average number of information bits per data symbol 
Table 3 The results from the simulated annealing optimization without using the PMW and PFD approach

\begin{tabular}{|c|c|c|c|c|c|}
\hline $\mathrm{BER}_{T}$ & $\mathrm{BER}_{M}$ & Symbol rep. & Conv. code & Avg. info. bits & Spectral eff \\
\hline $10^{-5}$ & $3.95 \times 10^{-1}$ & None & None & 3.12 & 3.12 \\
\hline $10^{-4}$ & $3.78 \times 10^{-1}$ & None & None & 3.25 & 3.25 \\
\hline $10^{-3}$ & $3.71 \times 10^{-1}$ & None & None & 3.19 & 3.19 \\
\hline $10^{-2}$ & $3.75 \times 10^{-1}$ & None & None & 3.12 & 3.12 \\
\hline $10^{-1}$ & $9.14 \times 10^{-2}$ & None & $\frac{1}{8}$ & 2.88 & 0.36 \\
\hline
\end{tabular}

Avg. info. bits is the average number of information bits per data symbol

implement the DFT. When $N=1024$ and $N_{\mathrm{CP}}=N / 4$, $N+N_{\mathrm{CP}}=1280$ and can be factored into the relative primes of 256 and 5. Similarly, when $N=1024$ and $N_{C P}=$ $N / 2, N+N_{C P}=1536$ and can be factored into the relative primes of 512 and 3. Although a closed-form expression does not exist for the complexity of the PFA, [36] lists the number of complex multiplies required for various transform sizes between 30 and 2520. From the given results, it can be generally stated that for large transform sizes, $N>128$, the number of complex multiplies required by the PFA for a transform of size $3 N / 2$ requires about the same number of complex multiplies as the Radix-2 FFT of transform size $N$. The use of the PFA does not result in a substantial increase in complexity for a single transform and, in some circumstances, may decrease it.

The number of complex multiplies needed to implement the Radix-2 N-point FFT is [37]:

$$
\frac{N}{2} \log _{2}(N)
$$

With the simplifying assumption that the PFA algorithm can implement the $N+N_{\text {CP }}$ sized DFT with approximately the same number of multiplies as the Radix- $2 N$-point $\mathrm{FFT}$, the relative increase complexity is approximately 3 times the number of multiplies required.

\subsubsection{Complexity of filtering}

Any FRESH filter, including the PFD, is able to exploit the cycle frequencies within the desired signal and any interference, described by (31) and (32) of (30). Each frequency bin is now the summation of multiple complex weighted bins equivalent to the number of cycle frequencies over $B$ OFDM symbols. The redundant bins can be utilized to our advantage by avoiding duplicate work and only computing the summation of correlated bins for a set of cycle frequencies one time.

The maximum number of complex multiplies needed to execute the PFD is:

$$
\left(N+N_{C P}\right)\left(\sum_{c=0}^{B-1} \sum_{b=0}^{B-1}\left(U_{c, b}+V_{c, b}\right)\right) .
$$

However, only $N+N_{c p}$ of the frequency bins need to be estimated due to the redundancies built into the signal. A reasonable simplifying assumption is made that the number of cycle frequencies is consistent across all $B$ OFDM symbols:

$$
\begin{array}{r}
U=U_{c, b} \forall c=0,1, \ldots, B-1 \\
b=0,1, \ldots, B-1, \\
V=U_{c, b} \forall c=0,1, \ldots, B-1 \\
b=0,1, \ldots, B-1 .
\end{array}
$$

The PFD therefore requires:

$$
\left(N+N_{\mathrm{CP}}\right)(U+V) \text {, }
$$

multiplies to weight the redundant OFDM symbols and:

$$
\left(N+N_{\mathrm{CP}}\right)(U+V) B,
$$

additions to perform the summation across the B OFDM symbols. The relative increase in complexity over the onetap equalization to implement the filtering is therefore:

$$
\frac{\left(N+N_{\mathrm{CP}}\right)}{N}(U+V),
$$

complex multiplies and:

$$
\frac{\left(N+N_{C P}\right)}{N}(U+V) B
$$

complex additions. The relative complexity increase of (55) can be separate into two terms: $\frac{N+N_{\mathrm{CP}}}{N}$ due to the larger FFT size and $U+V$ due to the exploitation of the spectral redundancies and conjugate spectral redundancies. The one-tap equalization uses $N_{C P}=0$ since it discards the cyclic prefix before the FFT, and $U=1$ and $V=0$ because it does not exploit any spectral redundancies.

An example is provided to demonstrate the increase in complexity in using the PFD, or any FRESH filter, relative to the one-tap equalizer. In this example, the PMW transmits QPSK data symbols which have been repeated twice, represented by 2 cycle frequencies, and the 16QAM interferer of Section 3.3 uses 3 frequencies in (29); therefore, $U=5$. Neither of the two signals have conjugate spectral redundancy; therefore, $V=0$. When $N_{\mathrm{CP}}=$ $\frac{N}{4}$, then $\frac{N+N_{C P}}{N}=\frac{5}{4}$. The relative increase in number of complex multiplies is therefore $\frac{5}{4} 5=6.25$, representative of all FRESH filters, including the PFD. The number of complex additions is increased by $6.25 B$. 


\section{Conclusions}

A novel method has been proposed for creating interference mitigation properties in paramorphic multicarrier waveforms to enable communication in cyclostationary interference limited environments. Spectral redundancy is formed through repeating data symbols in both time and frequency within OFDM symbols, which is exploited at the receiver for a signal processing gain. The method provides a new way to design signals to be robust against cyclostationary interference, while simultaneously producing a better BER than ECCs for similar overhead rates under the simulated scenarios.

Future work would include using more generalized repetition patterns, both varying the amount of repetition per symbol and its placement in time and frequency. The spectral redundancy could also be designed across multiple antennas, including the development of the appropriate array processing algorithms to exploit the spatially distributed spectral redundancy. A real-time cognitive engine could also be developed which takes into account the proposed waveform adaptation techniques.

\section{Endnote}

${ }^{1}$ The property of changing from one mineral species to another (as from aragonite to calcite) by a change in internal structure and physical characters but not in chemical composition. "Paramorphism", Def., MerriamWebster, http://www.merriam-webster.com/dictionary/ paramorphism

\section{Abbreviations \\ BER: Bit error rate; ECC: Error correcting code; CS: Cyclostationary signals; CF: Cycle frequency; DVB-S2: Digital video broadcasting second generation; FS: Frequency shift (within context of the operation); FRESH: Frequency shift (within context of the filtering structure); LDPC: Low density parity check; MMSE: Minimum mean squared error; MRC: Maximal ratio combining; PMW: Paramorphic multicarrier waveform; PFD: Paramorphic FRESH demodulator; SINR: Signal to interference and noise ratio; SNR: Signal to noise ratio; SIR: Signal to interference ratio; TV-FRESH: Time-varying FRESH}

\section{Funding}

The work of M. Carrick and J. H. Reed was supported in part by the National Science Foundation under grant CNS-1564148.

\section{Authors' contributions}

$\mathrm{MC}$ is the primary author of this paper, who developed the simulations and wrote the first draft of the paper. JHR has acted as his academic advisor through the duration, providing feedback at all levels. CMS provided substantial feedback on the whole manuscript, with a particular emphasis on the cyclostationary elements of the paper. All authors read and approved the final manuscript.

\section{Competing interests}

A patent has been submitted by M. Carrick and J. H. Reed covering materials in this manuscript, "Method for jointly adapting an OFDM waveform and the demodulator for interference mitigation and harsh channels," Patent ID: 62144039

\section{Publisher's Note}

Springer Nature remains neutral with regard to jurisdictional claims in published maps and institutional affiliations.

\author{
Author details \\ ${ }^{1}$ Bradley Department of Electrical and Computer Engineering, Virginia \\ Polytechnic and State University, Blacksburg, Virginia, 24061, USA. ${ }^{2}$ NorthWest \\ Research Associates, Monterey, California 93940, USA.
}

Received: 1 August 2017 Accepted: 18 December 2017

Published online: 05 January 2018

References

1. J Proakis, Digital Communications, 5th Edition. (McGraw Hill, New York, 2007)

2. WA Gardner, Cyclic Wiener filtering: theory and method. IEEE Trans. Commun. 41(1), 151-163 (1993)

3. S Haykin, Adaptive Filter Theory. (Prentice-Hall, Inc., Englewood Cliffs, N.J. 2001)

4. V Aue, JH Reed, in IEEE Forty-Fourth Vehicular Technology Conference. An interference robust CDMA demodulator that uses spectral correlation properties (IEEE, 1994), pp. 563-567

5. P Petrus, JH Reed, Time dependent adaptive arrays. IEEE Signal Process. Lett. 2(12), 219-222 (1995)

6. WA Gardner, A Napolitano, L Paura, Cyclostationarity: half a century of research. Signal Process. 86(4), 639-697 (2006)

7. A Napolitano, Cyclostationarity: new trends and applications. Signal Process. 120, 385-408 (2016)

8. W Gardner, L Franks, Characterization of cyclostationary random signal processes. IEEE Trans. Inf. Theory. 21(1), 4-14 (1975)

9. E Ferrara, Frequency-domain implementations of periodically time-varying filters. IEEE Trans. Acoust. Speech Signal Process. 33(4), 883-892 (1985)

10. JH Reed, TC Hsia, The performance of time-dependent adaptive filters for interference rejection. IEEE Trans. Acoust. Speech Signal Process. 38(8), 1373-1385 (1990)

11. JH Reed, NM Yuen, TC Hsia, An optimal receiver using a time-dependent adaptive filter. IEEE Trans. Commun. 43(2/3/4), 187-190 (1995)

12. K Maeda, A Benjebbour, T Asai, T Furuno, T Ohya, Cyclostationarity-inducing transmission methods for recognition among OFDM-based systems. EURASIP J. Wireless Commun. Netw. Cognitive Radio Dynamic Spectrum Sharing Syst. 2008(23), 1-14 (2008)

13. PD Sutton, KE Nolan, LE Doyle, Cyclostationary signatures in practical cognitive radio applications. IEEE J. Selected Areas Commun. 26(1), 13-24 (2008)

14. J Sun, D Qu, T Jiang, G Zhong, J Guo, in 2011 IEEE Vehicular Technology Conference (VTC Fall). Low overhead cyclostationary signatures based on hopping subcarrier in OFDM-based dynamic spectrum access networks (IEEE, 2011), pp. 1-5

15. P Sutton, Rendezvous and coordination in OFDM-based dynamic spectrum access networks.PhD thesis, University of Dublin (2008)

16. J Tian, $\mathrm{H} \mathrm{GuO}, \mathrm{H} \mathrm{Hu}, \mathrm{HH}$ Chen, Frequency-shift filtering for OFDM systems and its performance analysis. IEEE Syst. J. 5(3), 314-320 (2011)

17. N Shlezinger, R Dabora, Frequency-shift filtering for OFDM signal recovery in narrowband power line communications. IEEE Trans. Commun. 62(4), 1283-1295 (2014)

18. M Carrick, JH Reed, F harris, in 2017 IEEE International Conference on Digital Signal Processing (DSP). An optimal filter for signals with time-varying cyclostationary statistics (IEEE, 2017), pp. 1-5

19. M Carrick, Reed JH, in 2017 IEEE 38th Sarnoff Symposium. Improved GFDM equalization in severe frequency selective fading (IEEE, 2017), pp. 1-6

20. M Carrick, JH Reed, in 2017 IEEE Global Conference on Signal and Information Processing (GlobalSIP). Exploiting the cyclostationarity of radar chirp signals with time-varying filters (IEEE, 2017), pp. 1-4

21. WA Gardner, CM Spooner, Signal interception: performance advantages of cyclic-feature detectors. IEEE Trans. Commun. 40(1), 149-159 (1992)

22. S Kirkpatrick, C Gelatt, M Vecchi, Optimization by simulated annealing. Science. 220(4598), 671-680 (1983)

23. A He, KK Bae, TR Newman, J Gaeddert, K Kim, R Menon, L Morales-Tirado, J Neel, Y Zhao, JH Reed, WH Tranter, A survey of artificial intelligence for cognitive radios. IEEE Trans. Vehicular Technol. 59(4), 1578-1592 (2010)

24. E Serpedin, GB Giannakis, Blind channel identification and equalization with modulation-induced cyclostationarity. IEEE Trans. Signal Process. 46(7), 1930-1944 (1998)

25. H Bolcskei, Blind estimation of symbol timing and carrier frequency offset in wireless ofdm systems. IEEE Trans. Commun. 49(6), 988-999 (2001) 
26. W Gardner, Spectral correlation of modulated signals: part I—analog modulation. IEEE Trans. Commun. 35(6), 584-594 (1987)

27. OAY Ojeda, J Grajal, Adaptive-fresh filters for compensation of cycle-frequency errors. IEEE Trans. Signal Process. 58(1), 1-10 (2010)

28. WA Gardner, Statistical Spectral Analysis, A Nonprobabilistic theory. (Prentice-Hall, Inc., Englewood Cliffs, 1988)

29. WA Gardner, WA Brown, in Twenty-Third Asilomar Conference on Signals, Systems and Computers, 1989. Frequency-shift filtering theory for adaptive co-channel interference removal. vol. 2 (IEEE, 1989), pp. 562-567

30. J Zhang, KM Wong, ZQ Luo, PC Ching, Blind adaptive fresh filtering for signal extraction. IEEE Trans. Signal Process. 47(5), 1397-1402 (1999)

31. TC Clancy, M Norton, M Lichtman, in MILCOM 2013-2013 IEEE Military Communications Conference. Security challenges with LTE-advanced systems and military spectrum (IEEE, 2013), pp. 375-381

32. T Newman, Multiple objective fitness functions for cognitive radio adaptation.PhD thesis, University of Kansas (2008)

33. F Guidolin, Nekovee M, in 2015 IEEE Globecom Workshops (GC Wkshps). Investigating spectrum sharing between $5 \mathrm{~g}$ millimeter wave networks and fixed satellite systems (IEEE, 2015), pp. 1-7

34. European Telecommunications Standards Institute. Digital Video Broadcasting (DVB); second generation framing structure, channel coding and modulation systems for broadcasting, interactive services, news gathering and other broadband satellite applications (dvb-s2). ETSI Standard EN 302 307 V1.1.1 (2005)

35. TWada, in 2004 IEEE International Conference on Communications (IEEE Cat.No.04CH37577). A study on performance of LDPC codes on power line communications. vol. 1 (IEEE, 2004), pp. 109-113

36. D Kolba, T Parks, A prime factor FFT algorithm using high-speed convolution. IEEE Trans. Acoust. Speech Signal Process. 25(4), 281-294 (1977)

37. J Proakis, Digital Signal Processing, 4th Edition. (Pearson, London, 2006)

\section{Submit your manuscript to a SpringerOpen ${ }^{\circ}$ journal and benefit from:}

- Convenient online submission

- Rigorous peer review

- Open access: articles freely available online

- High visibility within the field

- Retaining the copyright to your article

Submit your next manuscript at $\gg$ springeropen.com 